\title{
IV. Das Wirtschaftsprogramm der NSDAP von 1932
}

\section{Die Reichstagsrede vom 10. Mai 1932}

In der Sitzung des Reichstages vom 10. Mai 1932 griff zum ersten Mal seit eineinhalb Jahren Gregor Straßer wieder als Redner in die Debatte ein. ${ }^{1}$ In einer grundsätzlichen Erklärung legte er ein umfassendes nationalsozialistisches Arbeitsbeschaffungs- und Krisenbekämpfungsprogramm vor. Im Vergleich zu dem damals insbesondere von den Nationalsozialisten geübten Stil fiel diese Rede durch ihre gemäBigte, gelegentlich geradezu verbindlich-kooperative Form auf. Vor allem aber die Sachlichkeit, mit der, vor wenigen demagogischen Schlenkern abgesehen, sowohl bekannte wie auch wesentlich neue Ideen zur Behebung der wirtschaftlichen Notlage vorgetragen wurden, sicherte der Rede breites Interesse weit über die NSDAP hinaus. Die Möglichkeit von Reichstagsneuwahlen war bereits im Gespräch, ${ }^{2}$ und Straßers Ausführungen konnten daher schon als die Basis der NSDAP für einen Wahlkampf gelten.

$\mathrm{Zu}$ Beginn seiner Rede wandte er sich scharf gegen die Deflationspolitik, die Brüning mit dem „Kampfmittel der Notverordnung" betreibe, was zwar einerseits die Notlage als solche aufzeige, aber ,,auf der anderen Seite Not verordnet.“3 Den Aufstieg der eigenen Partei, wie er sich in den Wahlen der letzten Zeit und in den steigenden Mitgliederzahlen zeigte, deutete er als den „Protest eines Volkes gegen einen Staat ..., der eine Wirtschaftsordnung erlaubt und möglich macht, die den Ertragsreichtum der Natur verfälscht, Weizen verbrennt, Kaffee ins Meer wirft, notwendige Lebensgüter in sinnloser Weise aufstapelt, ... Wenn der Verteilungsapparat des weltwirtschaftlichen Systems von heute es nicht versteht, den Ertragsreichtum der Natur richtig zu verteilen, dann ist dieses System falsch und muß geändert werden." Er fuhr fort: „Interessant und wertvoll an dieser Entwicklung (des Volksprotestes) ist die große antikapitalistische Sehnsucht .., die durch unser Volk geht, die heute schon 95 Prozent unseres Volkes bewußt und unbewußt erfaßt hat. Diese antikapitalistische Sehnsucht ist ... der Protest des Volkes gegen eine entartete Wirtschaft, und sie verlangt vom Staat, daß er, um das eigene Lebensrecht zu sichern, mit dem Dämon Gold, Weltwirtschaft, Materialismus, mit dem Denken in Ausfuhrstatistik und Reichsbankdiskont bricht und ehrliches Auskommen für ehrlich geleistete Arbeit wiederherzustellen in der Lage ist." Der SPD warf er vor, daß ihr zur Steuerung der Arbeitslosigkeit nichts anderes mehr einfalle als Arbeitszeitverkürzungen ohne Lohnausgleich. Arbeitszeitverkürzungen ohne Lohnausgleich seien aber nichts anderes als ein 16prozentiger Lohnabbau für alle noch Beschäftig-

1 Verhandlungen des Reichstages, Bd. 446, S. $2510 \mathrm{ff}$.

${ }^{2}$ Heiber, Goebbels-Tagebuch, S. 93.

3 Verhandlungen des Reichstages, Bd. 446, S. 2510 c.

${ }^{4}$ Ebenda, S. 2511 a, b, c. 
ten; laut Statistik seien durch verbreitete Kurzarbeit diese Verkürzungen samt den dadurch entstehenden Einkommensverlusten ohnehin schon längst Wirklichkeit geworden. Das Unsinnigste an solchen Maßnahmen zur Krisenbekämpfung sei aber, daß sie keinen Einfluß auf die Gesamtwirtschaft hätten, denn ,,die gesamte Kaufkraft wird nicht um einen Pfennig erhöht und damit nicht um einen Groschen neue Arbeit und Arbeitsmöglichkeit geschaffen. "5 Auf eben solche Nachfrageerhöhung komme es jedoch an, wobei Straßer glaubte, daß durch eine Vermehrung der Nachfrage nur ein kumulativer Wirtschaftsprozeß in Gang gesetzt werden müsse. „Ich bin felsenfest überzeugt davon, daß es überhaupt nur darum geht, einmal den Motor in Gang zu bringen. Arbeit erzeugt weitere Arbeit. Wir brauchen in diese Dinge gar nicht allzu sehr staatlich hinein zu dirigieren." $\mathrm{Da}$ aber angesichts der darniederliegenden Wirtschaft und auch der Größe des finanziellen Impulses kein privatwirtschaftliches Unternehmen hierzu bereit und in der Lage sei, betonte er: „Aber wir müssen die ersten großen Arbeitsbeschaffungsaufträge vom Staate her machen ... Wenn die großen Arbeitsaufträge angekurbelt werden, dann bin ich überzeugt, daß wir in zwei Jahren einen normalen Ablauf der Dinge automatisch wieder haben werden." 6 Für solche staatlichen Arbeitsbeschaffungsaufträge sah er folgende Möglichkeiten:

1. Landeskulturarbeiten, ${ }^{7}$ nämlich Entwässerung schlechter Böden oder von Mooren, Bodenverbesserung anderer Art, z. B. durch Kunstdüngung, Ödlandkultivierung, Flurbereinigung, Flußregulierung, Eindeichungen usw.

2. Eigenheimbau ${ }^{8}$ für Arbeiter zur Auflockerung der Mietskasernenviertel der Großstädte, was zusätzliche Wirtschaftsstätten liefere, wenn jeder etwa 1 Morgen zur Bebauung hinzubekomme.

3. Straßen- und Wegebau, Dammanlagen und Kanalisierungen zur Vermeidung von Hochwasserschäden, Binnenschiffahrtswege, ${ }^{9}$ Ausbau der Energiewirtschaft, Wiederinstandsetzung von Wohnungen. ${ }^{10}$

Das eigentliche Problem war natürlich nicht so sehr die Aufstellung eines Programms notwendiger und auch nützlicher Arbeitsvorhaben, sondern die Finanzierung solcher Maßnahmen. Zur Finanzierung aber sagte Straßer: Da alle diese Arbeiten sehr lohnintensiv seien und in den benötigten Materialien ebenfalls hohe Lohnkosten steckten, seien etwa $30 \%$ der benötigten Summe aus der dann eingesparten Arbeitslosenunterstützung aufzubringen, weitere $5 \%$ aus den wieder stärker fließenden Arbeitslosenversicherungsbeiträgen. $\mathrm{Da}$ bei belebter Wirtschaft auch die Steuereingänge wieder höher sein würden, könnten hieraus weitere $15 \%$ und damit insgesamt schon 50\% abgedeckt werden. Von den Nutznießern dieser Arbeiten, also

5 Ebenda, S. 2511d und 2512a, b.

6 Ebenda, S. 2516d.

7 Ebenda, S. $2515 \mathrm{dff}$.

8 Ebenda, S. $2514 \mathrm{df}$.

9 Ebenda, S. 2519c.

10 Rede im Berliner Sportpalast vor 15000 NSBO-Mitgliedern am 20.10.1932, auch als Flugblatt im Wahlkampf verwendet. 
den Bauern, deren Böden verbessert würden oder die Neuland gewönnen, und den Eigenheimerwerbern sollten etwa $20 \%$ der Kosten aufgebracht werden, dies sollte bei Straßen z. B. durch Auto- und Benzinsteuern geschehen und bei den Deichbauten durch den Wegfall der Kosten bei Hochwasserschäden. Es fehlten in seiner Berechnung noch 30\%. Der traditionelle Weg zu ihrer Deckung wäre eine In- oder Auslandsanleihe gewesen. Eine Auslandsanleihe, die damals an sich schwer bzw. nur zu politisch einengenden Bedingungen zu haben gewesen wäre, zog er nicht in Betracht. Eine Inlandsanleihe lehnte er jedoch entschieden ab, und zwar mit der Begründung, daß man erstens sowieso nichts erhalten und daß zweitens nur die Staatsverschuldung weiter zunehmen würden. Das Problem, daß bei einer Inlandsanleihe eventuell die Nachfrage lediglich von der privaten auf die staatliche Seite verschoben würde, ohne daß ein Wirtschaftsanstoß zustande käme, sah er offenbar nicht. Jedenfalls wollte er zur Aufbringung der in seinem Rechenbeispiel noch fehlenden $30 \%$ einen neuen Weg gehen. In diesem Gedanken ist das eigentlich interessante Element seines Wirtschaftsplans zu sehen. Um zusätzliche Nachfrage zu schaffen, wollte er jene Summe durch ,,produktive Kreditschöpfung“ gewinnen. Vor dem Reichstag glaubte Straßer dies nicht näher erläutern zu müssen, ${ }^{11}$ doch führte er die Idee am 20. Oktober 1932 vor 15000 NSBO-Mitgliedern im Berliner Sportpalast näher aus:

„Die Krediterweiterung geschieht aufgrund eines Kredites an das Reich durch die Reichsbank und die der Staatshoheit unterstellten Banken. Zur Durchführung dieser Krediterweiterung ist eine Vermehrung des Umlaufgeldes nur im Ausmaße von vielleicht 300 bis 400 Millionen RM erforderlich. Im übrigen vollzieht sich die Krediterweiterung auf dem Wege des Giralgeldes, das auch staatliches Geld ist und als solches in die staatliche Geldhoheit und das neu zu schaffende staatliche Geldund Kreditsystem mit einzubeziehen ist. Der Produktionswechsel und der Staatskredit werden zur Unterlage des Geldumlaufes. “12

Er war sich offenbar im klaren, daß er sich hier auf ein juristisch und auch psychologisch gefährliches Gebiet begab. Zum einen gab es gesetzliche Bestimmungen, die die Rentenmark an eine bestimmte Golddeckung banden, was durch außen- d. h. reparationspolitische Probleme kompliziert wurde, zum anderen war die Erinnerung an die Jahre 1922/23 noch so frisch, daß alle Maßnahmen, die den Geldumlauf erhöhen mußten, die Angst vor einer neuen Inflation zu wecken drohten. Wenn Straßer den Geldumlauf verändern wollte, mußte er mit der Reichsbank und den Anhängern der Golddeckung der Währung aneinandergeraten. Er forderte denn auch die sofortige Lösung vom Goldstandard für die deutsche Währung. Diese Forderung, die in Deutschland schon Tradition hatte, versuchte er durch das Urteil zweier anerkannter ausländischer Nationalökonomen, nämlich Cassel ${ }^{13}$ und Irving Fisher, ${ }^{14}$ abzusichern, die er folgendermaßen zitierte:

11 Verhandlungen des Reichstages Bd. 446, S. 2519a.

12 Rede im Berliner Sportpalast.

13 Cassel, Gustav, schwed. Finanztheoretiker, 1866-1945.

14 Fisher, Irving, amerik. Nationalökonom, 1867-1947. 
„So hat Herr Cassel vor kurzem schon geschrieben: ,Verzicht auf Goldwährung ist etwas grundsätzlich anderes als Inflation. Die Tatsache, daß sich ein Staat von der Goldwährung abwendet, bedeutet durchaus noch nicht, daß er damit die Inflation entfesselt und den Binnenwert seiner Währung heruntersetzt.'

Der amerikanische Nationalökonom Erwin Fischer ${ }^{15}$ schreibt: ,Die Idee, daß das Gold zu unserem Standard gewählt worden ist, weil es nach seinem Wert stabil sei, ist ein Mythos'.“

Ferner berief er sich auf das Institut für Konjunktur (Leiter Prof. Wagemann), das auf das Beispiel Englands hingewiesen habe, wo nach der Lösung vom Goldstandard zwar der zwischenstaatliche Wert des Pfundes auf etwa 70\% abgerutscht, die innerstaatliche Kaufkraft aber unverändert geblieben sei. ${ }^{16}$

Schwieriger war es, der allgemeinen Furcht vor eventuell inflationsfördernden Maßnahmen zu begegnen, wobei Straßer offensichtlich, ebenso wie die Allgemeinheit, übersah, daß bei dem 1932 deutlich erniedrigten Preisniveau ${ }^{17}$ auf keinen Fall von einer Inflation gesprochen werden konnte, ehe nicht die Preise die Höhe des Pegels von 1929, dem letzten Jahr vor der Weltwirtschaftskrise, überschritten. Selbst noch unter dem Schock der Inflation stehend, hatte Straßer in diesem Punkt offenbar noch Bedenken gegen seinen eigenen Plan. Er forderte daher staatliche oder genossenschaftliche Monopole und staatlich kontrollierte Festpreise, um einem Ausufern der Preise begegnen zu können. ${ }^{18}$ Außerdem riet er, mit der Kreditschöpfung vorsichtig umzugehen; der Staat sei, sobald eine „gefährliche Entwicklung“ in Sicht komme, verpflichtet, sie ,zur rechten Zeit abzudrehen “. ${ }^{19}$ Auch wollte er die Förderung durch den Staat auf Projekte beschränken, die für das Wohl der Allgemeinheit sowohl notwendig als auch sinnvoll seien. Im Gegensatz zur „,kapitalistischen Rentabilitätsberechnung“, die nur danach frage, ,wieviel dem Geldgeber an Zinsen wieder in die Kasse zurückfließt“, wollte er das setzen, „was wir die nationalsozialistische Rentabilitätsberechnung nennen möchten, indem man die für solche Arbeiten aufgewendeten Geldmittel unter dem Gesichtspunkt der Nation sieht, indem man fragt, was für die wirtschaftlichen, kulturellen und sonstigen Belange des ganzen Volkes damit geleistet wird“". ${ }^{20}$ Im übrigen hielt Straßer zur Absicherung seiner Wirtschaftsreform, vor allem wegen des Abgehens vom Goldstandard, eine weitgehende Autarkie für notwendig. ${ }^{21} \mathrm{Er}$ forderte eine Binnenwirtschaft, bei der die

15 Verhandlungen des Reichstages, Bd. 446, S. $2513 \mathrm{c}$ und d. Hier steht allerdings ,,der amerikanische Nationalökonom Erwin Fischer". Es kann sich hier nur um einen Schreibfehler handeln. Auch in der Tonbandaufnahme ist deutlich die englische Aussprache „Irving“ zu verstehen (Deutsches Rundfunkarchiv Frankfurt, Tonband Nr. VII, 20004/64).

16 Wirtschaftliches Sofortprogramm S. 10f.

17 Gerhard Kroll, Von der Weltwirtschaftskrise zur Staatskonjunktur, Berlin 1958, S. 90 ff.

18 Verhandlungen des Reichstages, Bd. 446, S. 2517 d.

19 Ebenda, S. 2519a.

20 Ebenda, S. 2519 b, c.

21 Zur Bedeutung des Wortes Autarkie siehe Otto Brunner, Werner Conze, Reinhart Kosellek, Geschichtliche Grundbegriffe, Bd. I, Stuttgart 1972, S. 377 ff. 
Einfuhr der Rohstoffe und die zu ihrer Bezahlung nötigen Exporte nach der Lösung vom Goldstandard durch eine Abwertung erleichtert werden müßten; die Abwertung wiederum sollte von einer Devisenkontrolle begleitet werden. ${ }^{22}$

Nachdem er dem Reichskanzler Brüning noch besten Willen bescheinigt, aber darauf hingewiesen hatte, daß es für Brüning unmöglich sei, die Probleme zu lösen, solange er sich einerseits auf eine mühsam zusammengehaltene Regierung „heterogener Parteien“ und andererseits auf die falschen „Wirtschaftsführer" stütze, beanspruchte er aufgrund der letzten Wahlergebnisse die Regierung für die NSDAP, die dann die notwendige Reform durchführen werde, und er schloß: „Die Erfüllung gebe uns der Herrgott, und das Urteil überlassen wir der Geschichte." 23

Alle nachfolgenden Redner in der Reichstagssitzung gingen auf Straßers Ausführungen bis in Einzelheiten ein. ${ }^{24}$ Reichskanzler Brüning stimmte Straßers Forderungen weitgehend zu und verwies auf in Vorbereitung befindliche ähnliche Maßnahmen seiner Regierung. ${ }^{25}$ Auch Hilferding, der Wirtschaftsexperte der SPD, und Heuß, der Vertreter der Staatspartei, nahmen die einzelnen Forderungen unter die Lupe und gaben Straßer in wesentlichen Punkten recht, wobei sie allerdings nachzuweisen versuchten, daß andere diese Gedanken schon früher geäußert hätten. Allerdings lehnten sie alle einen Punkt von Straßers Plan ab, und zwar die Kreditschöpfung, die Inflation bedeutete. Ohne ihre Behauptung näher begründen zu können, wandten sie sich damit gegen den wichtigsten Punkt dieses konjunkturpolitischen Planes. Es stellt sich die entscheidende Frage, wer hier recht hatte. War eine aktive Konjunkturpolitik überhaupt wünschenswert und durch eine staatlich gelenkte Belebung der Nachfrage, d. h. durch Kreditschöpfung zugunsten arbeitsintensiver Maßnahmen, überhaupt möglich?

\section{Konjunkturtheoretische Betrachtungen}

In der historischen Betrachtung muß zunächst das damals, im Jahre 1932, als gesichert angesehene Fachwissen berücksichtigt werden. Mit ihrer Ablehnung einer aktiven Konjunkturpolitik, die mit Kreditschöpfungen arbeiten sollte, befanden sich Brüning, sein Reichsbankpräsident Luther und viele andere Politiker in weitgehender Übereinstimmung mit den herrschenden Anschauungen der damaligen Zeit. ${ }^{26}$

22 Sofortprogramm S. 8 f.

23 Verhandlungen des Reichstages, Bd. 446, S. $2521 \mathrm{a}$.

24 Ebenda, S. $2521 \mathrm{ff}$; Brüning, Memoiren, S. 587, Heuss, Erinnerungen, S. $385 \mathrm{ff}$.

25 Siehe unten S. $117 \mathrm{f}$.

26 Horst Sanmann, Deutsche Wirtschafts- und Finanzpolitik in der Ära Brüning, in: Hamburger Jahrbuch für Wirtschafts- und Gesellschaftspolitik 10 (1965), S. 109-140, hier S. 110, 119f.; Henning Köhler, Arbeitsbeschaffung, Siedlung und Reparationen in der Schlußphase der Regierung Brüning, in: VfZ 17 (1969), S. 276; Ferdinand A. Hermens, Das Kabinett Brüning und die große Depression, in: „Staat, Wirtschaft und Politik in der Weimarer Republik“, Berlin 1967, S. 287 ff., hier besonders 303; Kroll, a. a. O., S. 175, 
So urteilte etwa der bekannte Nationalökonom Adolf Weber in seinem Lehrbuch „Allgemeine Volkswirtschaftslehre“, das 1929 erschien, ${ }^{27}$ „eine allgemeine Überproduktion (sei) unmöglich", ${ }^{28}$ womit er sich auf das Theorem berief, das Jean Baptiste Say 1803 so formuliert hatte: „Das Gesamtangebot an Gütern und die Gesamtnachfrage nach Gütern halten sich notwendigerweise die Waage, denn die Gesamtnachfrage ist weiter nichts als die Gesamtmenge der erzeugten Güter." ${ }^{29}$ Anders ausgedrückt: die Gesamtkosten der Produktion einer Volkswirtschaft, d. h. der Preis des Gesamtangebots, sind gleich der Gesamtnachfrage nach dieser Produktion, woraus folgt, daß sich Angebot und Nachfrage im ganzen immer von selbst schnellstmöglich ausgleichen. Frühe Kritiker, wie etwa Malthus, Rodbertus oder Marx, die an die Möglichkeit einer Úberproduktion glaubten, wurden von Weber beiseite geschoben, da es eine Überproduktion nur scheinbar und nur wegen kurzzeitiger Disproportionalitäten geben könne. ${ }^{30}$ Gerade solche Disproportionalitäten, nach Ansicht der ökonomischen Lehre Auslöser der Krisenerscheinungen, würden aber vor allem durch staatliche Eingriffe hervorgerufen oder zumindest verstärkt, weshalb sich der Staat nicht in den Konjunkturverlauf einmischen dürfe. Weber glaubte daher, daß die Schwankungen der Konjunktur am besten durch die Selbstheilungskräfte der liberalen Wirtschaft behoben würden, wobei er diesen Schwankungen auch noch die Funktion einer Selbstreinigung der Wirtschaft zuerkannte. So verurteilte er jede auf wirtschaftliche Stabilität gerichtete Politik, vollends aber die Idee einer aktiven Notenbankpolitik zur Steuerung der Konjunktur. Der Notenbank fiel seiner Ansicht nach nur die Aufgabe zu, ,die Kräfte der Selbstregulierung im wirtschaftlichen Leben zu erkennen ... Der Zins muß als freier Marktpreis fungieren können. Formal wird der für die Konjunkturgestaltung wichtigste Zins, der Diskontsatz, von oben her durch die Zentralnotenbank bestimmt. Aber er wird seiner Aufgabe nur dann gerecht werden können, wenn er nicht durch irgendeine Willkür, durch irgendein wirtschaftspolitisches Programm des jeweiligen Leiters der Zentralnotenbank, sondern durch die realen Geldmarktverhältnisse bestimmt wird.“ Einer anderen Notenbankpolitik warf er Merkantilismus vor, ${ }^{31}$ was für ihn, den liberalistischen Wirtschaftstheoretiker in der Nachfolge Says, die schlimmste Abqualifizierung bedeutete. Er zitierte Landsberg, wenn er schrieb: „Auf diese Weise findet ein

bemerkt: „Aus diesen Lehrbüchern der Nationalökonomie konnte ein deutscher Staatsmann in den Jahren 1930-32 nichts, aber auch gar nichts entnehmen, was auch nur im geringsten geeignet gewesen wäre, den Kampf mit der Weltwirtschaftskrise aufzunehmen, ein Tatbestand, den man doch wohl nicht gut anders, denn als Fiasko der Nationalökonomie bezeichnen kann!" Vgl. ferner Jörg Ohl, Keynesianismus ohne Keynes. Der deutsche Beitrag zur Entstehung der modernen Beschäftigungstheorie, Diss. jur. Tübingen 1953, S. 34.

27 Adolf Weber, Allgemeine Volkswirtschaftslehre, München und Leipzig 1929.

28 A. Weber, a. a. O., S. 418.

29 Traité, 1. Ausgabe II, S. 175, zitiert nach Gide und Rest, Geschichte der Volkswirtschaftlichen Lehrmeinungen, deutsch von R. W. Horn, Fischer 1923, S. 125.

30 A. Weber, a. a. O., S. $420 \mathrm{f}$.

31 Ebenda, S. 471. Tatsächlich berief sich Keynes später auf die Ideen des Merkantilismus. 
Absolutismus Eingang in die Wirtschaft, der durch das Bestimmungsrecht der Parlamente in keiner Weise gemildert wird ... Wenige Monarchen haben die Machtfülle

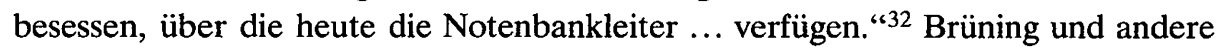
Politiker waren also bei ihrer Ablehnung der Straßerschen Vorschläge durch die anerkannte ökonomische Lehrmeinung gedeckt, was sich auch darin ausdrückte, daß die Grundzüge der Brüningschen Finanz- und Wirtschaftspolitik von den meisten ökonomischen Wissenschaftlern des In- und Auslandes bejaht wurde. ${ }^{33}$

Nun hat sich, unmittelbar durch die Weltwirtschaftskrise von 1929-33 angeregt, die Konjunkturforschung weiterentwickelt und wesentliche neue Erkenntnisse angesammelt. 1936 stellte Keynes in seiner „General Theory of Employment, Interest and Money“"34 der klassischen Theorie eine Theorie entgegen, mit der er die Grundlage für die moderne Konjunkturtheorie legte. ${ }^{35}$ Keynes legte dar, daß die ,klassische Theorie“ „nur in einem - praktisch nie erreichbaren - Sonder- und Grenzfall gültig“ ${ }^{36}$ sei. Er erkannte zwar an, daß das gesamte Einkommen áus dem Verkauf der Produktion stamme, negierte aber die Folgerung, daß damit alle Produktionskosten automatisch durch die Verkaufserlöse gedeckt seien. Um dies aufzuzeigen, wies er darauf hin, daß die Gesamtproduktion aus zwei grundsätzlich unterschiedlichen Produkten bestehe, und zwar 1. aus Verbrauchsgütern, 2. aus Investitionsgütern. Ein Teil des Gesamteinkommens einer Volkswirtschaft - bei niedrigem Einkommensniveau natürlich annähernd alles - werde für den Verbrauch bestimmt, der andere Teil nach einer dem Menschen eigentümlichen Verhaltensweise gespart, was Keynes als „Vorliebe zur Liquidität" bezeichnete. Hierbei könne unter Sparen natürlich Verschiedenes verstanden werden, z. B. Thesaurieren, Entschulden, Rücklagenbilden für eventuelle Notlagen oder später geplante Vorhaben. Wichtig sei nur, $\mathrm{da} ß$ diese Sparrate nicht notwendigerweise sofort wieder zur Nachfrage werde. Es entstehe also eine Lücke zwischen den Gesamtkosten der Produktion und der Gesamtnachfrage, und zwar in Höhe jener Sparrate. Die Lücke könne nur dadurch geschlossen werden, daß mit der Sparrate Investitionen gemacht, $d . h$. daß die produzierten Investitionsgüter gekauft würden. Während aber die Abnahme der Verbrauchsgüter eine im wesentlichen zwangsläufige sei, wobei Keynes allerdings auch hier noch im „Hang zum Verbrauch“ einen freiwilligen, von psychologischen Einflüssen regulierten Antrieb erkannte, unterliege das Einsetzen der Sparrate für Investitionen einer nahezu völlig freiwilligen Entscheidung. Der Entschluß der potentiellen Investoren, also der Unternehmer, ob sie investieren, hänge von den psycholo-

\footnotetext{
32 Ebenda, S. $471 \mathrm{f}$.

33 Siehe unten S. 117.

34 John Maynard Keynes, The General Theory of Employment, Interest and Money, London/New York 1936, deutsche Ausgabe, Berlin 1952.

35 Vgl. Walter Adolf Jöhr, Konjunktur, in: Handwörterbuch für Sozialwissenschaften, S. $97 \mathrm{ff}$.

36 Ernst Winkler, John Maynard Keynes und Silvio Gesell, in: Berichte der Gesellschaft für wirtschaftswissenschaftliche und soziologische Forschung, 1951 Heft 2, S. 7.
} 
gisch bedingten kurz- und langfristigen Zukunftshoffnungen $a b^{37}$ bzw., wie Keynes sagt, von den „Erwartungen“. Verbessert wurde dieses statische Modell Keynes” später durch die Einführung dynamischer Zeitfaktoren; hier sind vor allem Alvin Hansen ${ }^{38}$ sowie Zwiedeneck-Südenhorst ${ }^{39}$ und $\mathrm{Kroll}^{40}$ zu nennen.

Ohne weiter auf Keynes' Krisentheorie und die von ihm aufgestellten mathematischen Formeln, aus denen er seine „Allgemeine Theorie" ableitete, eingehen zu wollen, sei noch einmal das Wichtigste zusammengefaßt: Entscheidend für den Konjunkturverlauf ist nach Keynes folgendes Verhältnis: einerseits der „Hang zum Verbrauch“ und die auf positiven „Erwartungen“ beruhende ,Veranlassung zur Investition" und andererseits die „Vorliebe zur Liquidität", d. h. die Hortung der Sparrate. Ist die „Vorliebe zur Liquidität“ gleich Null, dann bedeutet dies, daß das gesamte Einkommen zum Verbrauch bzw. zur Investition verwandt wird, woraus folgt, daß die Gesamtproduktion Abnahme findet und die Konjunktur durchgehalten wird. Steigen der Verbrauch und die Investition über das Gesamteinkommen an, z. B. durch Kreditaufnahme, sinkt also die „Vorliebe zur Liquidität" unter Null, so steigt die Konjunktur an. Wenn allerdings die Geldhortung auf einen Wert über Null ansteigt, so fehlt dieser Betrag des Gesamteinkommens bei der Abnahme der Gesamtproduktion. Es kommt zur Absatzstockung, zur Produktionsschrumpfung, zum Konjunktureinbruch.

Es ist für Keynes daher klar, daß zur Behebung einer Wirtschaftskrise die Nachfrage mit jedem zur Verfügung stehenden Mittel gesteigert werden muß. Da der von massenpsychologischen Motiven ${ }^{41}$ gesteuerte Verbrauch nur schwer dahingehend beeinflußbar ist, seinen „Hang zum Verbrauch“ oder gar seine „Investitionsneigung“ auf Kosten seiner „Vorliebe zur Liquidität" zu steigern, sieht Keynes den staatlichen Eingriff als einzig gangbaren Weg. Er fordert mithin, daß der Staat in solchen Fällen in ausreichendem Maße selbst investieren muß, auch wenn die Investitionen vom wirtschaftlichen Standpunkt aus gesehen, verlustreich sind. Die Finan-

37 Keynes, a. a. O., S. 165 f.

38 Alvin Hansen, Richard Clemence, Reading in Business Cycles and National Income, New York 1953.

39 Otto v. Zwiedineck-Südenhorst, Die Arbeitslosigkeit und das Gesetz der zeitlichen Einkommensfolge, Weltw. Arch., Jena Bd. 34 (1931); vgl. auch Kroll, a. a. O., S. 301; Grotkopp, a. a. O., S. 280. Grotkopp weist darauf hin, daß Zwiedineck-Südenhorst einen gewissen Einfluß als wirtschaftspolitischer Berater, in den ersten Jahren nach der Machtübernahme, auf Hess und seinen Kreis hatte.

40 Kroll, Gerhard, Neutrales Geld und Kapitalbildung, Berlin 1935.

41 Jöhr, a. a. O., S. 111: ,Daß eine solche unmittelbare Beeinflussung von Mensch zu Mensch besteht und sich aus einem geringfügigen Anlaß eine epidemische Bewegung großer Stärke ergeben kann, ist von der Psychologie längst nachgewiesen ... Diese Beeinflussung, die als sozial-psychologische Infektion bezeichnet werden könnte, kann sich in ganz verschiedener Weise vollziehen: durch Motivübernahme, Bestreben anderen zuvorzukommen, Erweckung von Vorstellungen, Nachahmung, Übertragung von Gefühlen und Beeinflussung von Stimmungen ... Aufgrund dieser sozial-psychologischen Interdependenz ergibt sich eine Prädisposition für kumulative Prozesse, die auch für das Wirtschaftsleben von großer Bedeutung ist." 
zierung solcher Staatsinvestitionen muß aber so erfolgen, daß neue Nachfrage, also neues Geld auf dem Markt erscheint, was bedeutet, daß man einen Staatshaushalt zeitweilig nicht ausgleicht, also bewußt die Ausgabenseite entsprechend größer läßt als die momentanen Einnahmen, oder auf andere Art und Weise Geldschöpfung betreibt. Es ist dies in Keynesschen Worten die Forderung nach dem ,deficit spending" bzw. das, was heute unter antizyklischer Wirtschaftspolitik verstanden wird. Straßer hat nun im Frühjahr 1932, allerdings ohne Gründe für die inneren Ursachen des Konjunkturzusammenbruchs anzugeben und andererseits in der demagogischen Sprache des auf Publikumsgunst und Anhängerwerbung ausgehenden Politikers, doch die gleichen Prinzipien zur Überwindung der Krise verfochten wie Keynes. Seine ökonomische Hauptforderung war die Vermehrung der Nachfrage, ${ }^{42}$ die er durch arbeits- und lohnintensive Aufträge des Staates herbeiführen wollte; die Aufträge brauchten nicht unmittelbar rentabel zu sein. ${ }^{43}$ Zur Finanzierung solcher Maßnahmen zur Wirtschaftsankurbelung verlangte er Kreditschöpfung. ${ }^{44} \mathrm{Er}$ verstand darunter Kredite, die sich das Reich bei der Reichsbank holen und in Form von Girageld in den Wirtschaftskreislauf pumpen sollte, ${ }^{45}$ also genau jene antizyklische Wirtschaftspolitik, die Keynes mit seiner Forderung des „deficit spending“ gemeint hatte.

Im Gegensatz zu Straßer, der weder in der Rede vom 10. Mai, noch in späteren Äußerungen Angaben über die Entstehung seines Programms machte, gab Keynes einige interessante Hinweise auf seine Vorläufer. ${ }^{46}$ Keynes sagte, die grundlegende Erkenntnis von der entscheidenden Bedeutung der zu geringen Nachfrage sei bereits bei Malthus und Rodbertus vorhanden gewesen, aber seit damals durch die ,klassische Nationalökonomie" verdrängt worden. Bis er diese Grunderkenntnis wieder ans Licht gezogen habe, habe sie ,in den Unterwelten eines Karl Marx, Silvio Gesell und Major Douglas verstohlen fortgelebt. “47 Am erstaunlichsten ist, daß er in dieser Aufzählung den Namen Silvio Gesell erwähnte. ${ }^{48}$

Gesell war gegen Ende des 19. Jahrhunderts in Argentinien als Kaufmann zu einem ansehnlichen Vermögen gekommen, das es ihm hinfort erlaubte, seinen Interessen zu leben. Als Kaufmann hatte er in Argentinien auch mit Währungs- und Wirtschaftskrisen zu tun gehabt. Als Praktiker machte er sich seine Gedanken über deren Ursachen, was ihn auf die Idee einer Geld- und Wirtschaftsreform brachte. Nach Deutschland zurückgekehrt, widmete er sich im wesentlichen der Propagierung seiner Idee. In der Münchner Räterepublik wurde er im April 1919 für wenige Wochen

${ }^{42}$ Verhandlungen des Reichstages, Bd. 446 S. 2511 d und S. 2512a, b.

43 Ebenda, S. 2515a.

44 Ebenda, S. 2514c.

45 Rede im Berliner Sportpalast, S. 13.

$46 \mathrm{Vgl}$. hierzu auch Alvin H. Hansen und Richard V. Clemence, a. a. O.

47 Keynes, a. a. O., S. 28; vgl. Winkler, a. a. O., S. 12.

48 Silvio Gesell, geb. in St. Vith (Malmedy) 17.3.1862, gest. in Eden bei Oranienburg (Brandenburg) 11.3. 1930. Einzige Biographie W. Schmid, Silvio Gesell, Bern 1954. Hauptwerk: Natürliche Wirtschaftsordnung durch Freiland und Freigeld, 1911. 
zum Finanzminister ernannt. In seiner blumigen, unwissenschaftlichen Ausdrucksweise, deren er sich bei der Verbreitung seiner Pläne bediente, und in seinen romantizistischen sozialreformerischen Vorstellungen, die sich vorzugsweise, rückwärts gewandt, an agrarischen Strukturen orientierten, gingen seine Vorschläge zur Wirtschafts- und Währungsreform fast völlig unter, so daß er von der Wissenschaft nicht recht ernstgenommen wurde. Um so mehr muß es verwundern, daß Keynes ihn offensichtlich als wichtigen Vorläufer anerkannte. Keynes erwähnt Gesell öfter in seinem Hauptwerk. Einmal geht er sogar auf ihn ein, sozusagen um ihn zu rehabilitieren und um sich bei ihm zu entschuldigen: „Es ist zweckmäßig, an dieser Stelle den seltsamen, zu Unrecht übersehenen Propheten Silvio Gesell (1862-1930) zu erwähnen, dessen Werk Einfälle tiefer Einsicht enthält und der nur gerade verfehlte, bis zum Kern der Sache vorzudringen. In den Nachkriegsjahren (nach dem 1. Weltkrieg, d. Verf.) bombardierten mich seine Anhänger mit Exemplaren seiner Werke, aber wegen gewisser offenkundiger Mängel seiner Beweisführung verfehlte ich vollständig, ihre Vorzüge zu entdecken. Wie so oft im Fall unvollkommen analysierter Eingebungen wurde ihre Bedeutung erst augenscheinlich, nachdem ich meine eigenen Folgerungen auf meine eigene Art erreicht hatte. Wie andere akademische Ökonomen behandelte ich inzwischen seine tief originellen Bestrebungen als nichts Besseres als die eines Überspannten. Da die Bedeutung Gesells voraussichtlich wenigen Lesern dieses Buches sehr vertraut sein wird, will ich ihm einen sonst unverhältnismäßig großen Platz einräumen.“49

Beim Vergleich der Hauptwerke von Keynes und Gesell, der „General Theory of Employment, Interest and Money“ von 1936 und der ,Natürlichen Wirtschaftsordnung" von 1911, ist es leicht, eine große Anzahl von Übereinstimmungen vor allem dort zu erkennen, wo beide der „klassischen Ökonomie“ widersprechen. ${ }^{50} \mathrm{Jedoch}$ ist es schwierig, ihre theoretischen Grundlagen zu vergleichen, da sie eine völlig unterschiedliche Sprache sprechen. Auf der einen Seite die blumige Sprache des Kaufmanns Gesell, die nicht frei ist von logischen Fehlern und Inkonsequenzen, wie Keynes richtig bemerkte, auf der anderen Seite Keynes streng wissenschaftliche Betrachtungsweise, die nahezu alle wichtigen Denkprozesse in exakten mathematischen Formeln ausdrückt. Beide glauben aber, die Krisenursache in der mangelnden Nachfrage entdeckt zu haben, was beide mit falscher Anwendung des Geldeinkommens erklären. ${ }^{51}$ Die von Keynes als zentraler Punkt entdeckte „, Vorliebe zur Liquidität“" bezeichnet Gesell sinngemäß gleichartig als „Hortung von Bargeld“. ${ }^{52}$ Um dieser Erscheinung, die beide als eigentlich krisenauslösend betrachten, zu begegnen, will Keynes vor allem den Weg staatlicher Investitionen gehen. Hier setzt Gesell, wenn man so will, früher ein, indem er das Wesen des Geldes dergestalt verändern will, daß es sich nicht mehr lohnt, es zu horten. In einer für ihn typischen

49 Keynes, a. a. O., S. $298 \mathrm{ff}$.

50 Winkler, a. a. O., S. $6 \mathrm{f}$.

51 Ebenda, S. 13ff.

52 Ebenda, S. 15. 
Parabel um Robinson Crusoe - die auch Keynes ausdrücklich als gut bezeichnet ${ }^{53}$ legt er dar, ,daß in einer reinen Naturaltauschwirtschaft der Schuldner für entliehene Waren keinen Zins bezahlt, weil der Gläubiger dankbar ist, wenn ihm durch Rückerstattung des vollen Wertes die Durchhaltekosten abgenommen werden." Damit begründet er die „Ưberlegenheit des Geldes über die Ware“, da ja beim Horten des Geldes prinzipiell keine Durchhaltekosten entstehen; er meint hier das, was Keynes die „Liquiditätsprämie des Geldes“ nennt. Während sich aber Keynes mit dieser Eigenart des Geldes abfindet, will Gesell ein Geld in Umlauf bringen, das jährlich einen bestimmten Prozentsatz (etwa 4-5\%) seines Wertes verliert, also „rostet" oder „verdirbt" wie eine Ware. Als Effekt werde jede längere Hortung von Geld unrentabel, weshalb es möglichst sofort wieder als Nachfrage in Erscheinung trete. Keynes erkannte auch diesen Gedanken als ,gesund“ an und hielt es für möglich, ,daß Mittel gefunden werden könnten, um ihn in bescheidenem Rahmen in der Wirklichkeit anzuwenden". Indes sah er die bestehenden Schwierigkeiten für eine totale Verwirklichung als zu groß an. ${ }^{54}$

Wenn man die Theorien Gesells aus ihren romantisierenden Einkleidungen herauslöst, zeigt sich also überraschende UUbereinstimmung mit Keynes, sowohl hinsichtlich der Grundlagen wie hinsichtlich der geforderten Maßnahmen. Keynes ist, da er diese Übereinstimmung selbst nicht leugnete, sogar angegriffen worden; seine Gegner versuchten, indem sie ihm solche Vorläufer vorwarfen, seine ganze Theorie zu diskreditieren: „Dem Kenner der Dogmengeschichte der Nationalökonomie kann es nicht entgehen, daß diese Theorie, die sich so modern gebärdet und angeblich gegenüber den Klassikern einen so erheblichen Fortschritt darstellt, in Wirklichkeit in allen wesentlichen Zügen mit denjenigen Theorien übereinstimmt, die man die vorklassischen oder vorwissenschaftlichen Theorien nennt und deren endgültige Widerlegung als Großtat der Klassiker galt.

Keynes war sich der Tatsache bewußt, daß er Vorgänger hatte, wohl bewußt, zitiert und rühmt er doch ausdrücklich die Merkantilisten, John Law und von neueren den Freigelder Silvio Gesell und einige andere, deren Lehren als die Lehren von wissenschaftlichen Hochstaplern abgetan zu werden pflegen. Keynes hat diese Männer mit Recht zitiert. Denn alle diese Schriftsteller sind Keynesianer in den beiden wesentli-. chen Fragen: in der Frage nach den möglichen Ursachen von Deflation und in der Frage der Wirkung von In- und Deflation auf den Beschäftigungsgrad."55

53 Keynes, a. a. O., S. 301; Winkler, a. a. O., S. 27.

54 Keynes, a. a. O., S. 302.

55 Albert Hahn, Die Grundirrtümer in Lord Keynes' General Theory of Employment, Interest and Money, in: Ordo, Jahrbuch für die Ordnung von Wirtschaft und Gesellschaft, Band 2, 1949, S. 170-192, hier S. 177. 
Silvio Gesell war im Jahre 1900 aus Argentinien zurückgekommen und hatte sich in der Schweiz niedergelassen, um die neuen Ideen, die er in Südamerika gefunden hatte, weiter durchzudenken und zu verbreiten. Bereits 1902 gab er seine erste kleine Zeitschrift, die „,Geldreform“, heraus. ${ }^{56}$ Bald stieß er bei seinen Studien über die Geldreform auf die Frage der Bodenreform, angeregt durch die Lektüre der Werke Henry Georges. ${ }^{57}$ Er trat in Verbindung zu den deutschen Agrar- und Bodenreformern um Damaschke, ${ }^{58}$ aus deren Kreisen auch seine ersten Anhänger kamen. Die Forderungen nach einem seiner Hortfähigkeit entkleideten und vom Golde gelösten „Freigeld“" wie auch nach einem aus Privatbesitz befreiten „Freiland" blieben hinfort seine beiden Hauptziele. 1909 gründeten seine Anhänger in Berlin eine Vereinigung, den „Physiokratischen Kampfbund“, 59 und begannen mit Flugblättern, Schriften, Büchern und Eingaben alle möglichen Wirtschaftswissenschaftler, Politiker, Bankiers, Gewerkschaftsfunktionäre und Zeitungen zu „,bombardieren" ". ${ }^{60}$ Seit etwa 1910-1912 wurde die Anhängerschaft an verschiedenen Stellen in Deutschland zahlreicher, und hier und dort schlossen sich kleine Debattierclubs zusammen.

Genau zu dieser Zeit, im Jahre 1912, trat der bayerische Beamte Peter Straßer unter dem Pseudonym P. Wegr. anläßlich der „Reichs-Finanz-Reform“ mit einem 14 Seiten langen Traktat an die Öffentlichkeit. ${ }^{61}$ Er begründete seine Schrift mit dem „Zweifel an der Güte unserer wirtschaftlichen Verhältnisse, an der Weisheit unserer Regierungsmänner“, wodurch ,,das Erdreich bereitet" werde, ,um aufzunehmen die Saat des Neuen, Besseren, des Sozialismus" ". ${ }^{62}$ Er machte einige bibliographische Angaben, aus denen die Herkunft seiner Ideen erschlossen werden kann. So nannte er Professor Friedrich Kleinwächter ${ }^{63}$ und Michael Flürscheim, ${ }^{64}$ die beide als Agrarreformer die Forderung nach Gemeineigentum an Grund und Boden erhoben, ferner Professor Enrico Ferri, ${ }^{65}$ der damals sozialistischer Abgeordneter im italienischen Parlament war und als Wissenschaftler vor allem die Abhängigkeit der Krimi-

56 Werner Schmid, a. a. O., S. 69.

57 Ebenda, S. 72. Henry George, amerikanischer Nationalökonom, 1839-1897. Er forderte die Aufhebung des privaten Bodeneigentums und die Einziehung der Bodenrente oder des unverdienten Wertzuwachses durch Besteuerung desselben.

58 Schmid, a. a. O., S. $81 \mathrm{f}$.

59 Ebenda, S. 97, 107.

${ }^{60}$ Ebenda, S. 97, 102 f., 117; Keynes, a. a. O., S. 298.

61 Paul Wegr. (Peter Straßer), Das neue Wesen, Kempten 1912.

62 Ebenda, S. 3.

63 Ebenda, S. 5. Prof. Friedrich Kleinwächter, geb. 1838 in Prag, Nationalökonom.

${ }^{64}$ Ebenda, S. 5. Michael Flürscheim, 1844-1912, Industrieller und in zahlreichen Schriften Verfechter einer Verstaatlichung von Grund und Boden. Aus seinem Ideenkreis entstand 1888 der „Deutsche Bund für Bodenbesitzreform“.

65 Ebenda, S. 10. Prof. Enrico Ferri, 1856-1929, Strafrechtler; 1886-1919 sozialistischer Abgeordneter, trat 1926 zum italienischen Faschismus über. 
nalität von soziologischen Bedingungen lehrte. Besonders interessant ist im übrigen, daß er auch den volkswirtschaftlichen Schriftsteller Rudolph Meyer ${ }^{66}$ zitierte, der in engstem Kontakt zu Rodbertus stand und überdies engster sozialpolitischer Mitarbeiter Herrmann Wageners war, jenes Vertrauten Bismarcks, der die wichtigsten Vorarbeiten für dessen Sozialgesetzgebung geleistet hatte. ${ }^{67}$

Peter Straßer forderte als Grundlage eines Volksorganismus die gesunde Familie, die in ein christliches und ständisch gegliedertes Staatswesen eingeordnet ist. Zwei Haupthindernisse gab es seines Erachtens auf dem Wege dahin: Zum einen das private Eigentum an Grund und Boden, zum anderen die Úbermacht der kapitalistischen Großindustrie. Mit der Forderung der Überführung des Bodens ins Gemeineigentum - bei der Abwicklung wies er den Gemeinden die entscheidende Aufgabe zu - stand er ganz auf seiten des Bodenreformers Damaschke; die Radikalität, mit der er vorgehen wollte, außerdem die Finanzierung durch Staatsobligationen und ein Grundrentensystem erinnerten eher an die „Freiland“ Forderungen Gesells. Die Industrie wollte er ,vergesellschaften“, worunter er verstand, daß sie auf der Basis der bestehenden Berufsgenossenschaften in Gemeinbesitz überführt werden müßten. Obgleich er seine Schrift anläßlich der öffentlichen Diskussion über eine Reichsfinanzreform herausbrachte, wie er im Vorwort angab, ging er merkwürdigerweise im Text mit keinem Wort auf Währungs- und Finanzfragen ein, sodaß es in dieser Hinsicht unmöglich ist, sein Verhältnis zu Gesell zu überprüfen. Immerhin ist aus der Schrift zu erkennen, daß er mit seinen Überlegungen in der Nähe der Ideen Gesells stand, und das gerade zu dem Zeitpunkt, da Silvio Gesell in Deutschland bekannt zu werden begann. Auch aufgrund der Tatsache, daß beide sich an Damaschke anlehnten, ist als ziemlich sicher anzunehmen, daß Silvio Gesell für Peter Straßer damals kein Unbekannter war. ${ }^{68}$

Nun zeigt dieses Traktat eines niederbayerischen Kommunalbeamten, in welcher politisch-wirtschaftlichen Ideenwelt die drei Straßer-Söhne, Gregor, Paul und Otto, aufgewachsen sind, zumal bezeugt ist, daß sich Vater Straßer regelmäßig mit ihnen über solche Fragen zu unterhalten pflegte. ${ }^{69}$ Es war für die Söhne also nichts Ungewöhnliches, sich Gedanken über die Sozialisierung von Grund und Boden und auch der Industrie zu machen. Es kann ferner als wahrscheinlich gelten, daß Gregor und Otto bereits damals von den Ideen Gesells zur Geldreform gehört hatten. Diese Grundideen sollten die beiden politisch aktiv gewordenen Brüder niemals aufgeben. Ein zeitlich etwas näher liegender Einfluß auf Gregor Straßer ist in den wirtschafts-

66 Ebenda, S. 14. Rudolf Herrmann Meyer, 1839-1899, Journalist und Sozialpolitiker; Hrsg. der „Briefe und sozialpolit. Aufsätze“ von K. Rodbertus (2 Bde., 1882). Wegen seiner Schrift „Polit. Gründer und die Korruption in Deutschland“ (1877) zu Gefängnis verurteilt.

67 Wolfgang Saile, Herrmann Wagener und sein Verhältnis zu Bismarck, Tübingen 1958, S. 97-122, hier besonders S. 112.

68 Otto Straßer gibt an, er habe schon im Elternhaus durch seinen Vater den Namen Silvio Gesell gehört; Auskunft v. 10. 4. 1971.

69 Pater Bernhard Straßer, a. a. O., S. 3; Auskunft Otto Straßer v. 10. 4. 71. 
politischen Gedanken Rathenaus zu erkennen. Vor allem die Rathenausche Vorstellung von einem ,starken und reichen“ Staat, der die Wirtschaft zum Wohle aller lenkt, hat ihn besonders interessiert, ${ }^{70}$ wobei anzumerken ist, daß auch Rathenau in brieflicher Verbindung mit Gesell stand. ${ }^{71}$ Zwar ist nicht anzunehmen, daß diese Verbindung schon Einfluß auf das von Gregor Straßer gelesene Hauptwerk Rathenaus „Von kommenden Dingen“ genommen hat. Fest steht aber, daß Rathenau einer der wenigen Politiker gewesen ist, die sich zumindest nach dem Ersten Weltkrieg bereit fanden, sich mit Gesells Werk intensiv zu beschäftigen und sogar ein persönliches Treffen zu vereinbaren.

Gesell war 1919, und zwar durch Ernst Niekisch, der zu seinen Anhängern gehörte, als Finanzminister in die erste bayerische Räteregierung hineingezogen worden. ${ }^{72}$ Nach dem Ende der Räteherrschaft war er als Hochverräter vor Gericht gestellt, aber am 9. Juli 1919 freigesprochen worden. ${ }^{73}$ Unmittelbar danach hat er mit seinen Anhängern die Werbetätigkeit für seine Reformideen wieder aufgenommen. Von ihren Zeitschriften, Flugblättern und Büchern abgesehen, warben sie jetzt vornehmlich mit Vorträgen und auf Versammlungen. ${ }^{74}$ Doch gibt es nur wenige Angaben über die Zahl der Anwesenden. Auch ist nichts Genaueres über die Zusammensetzung der Zuhörer und ihre Reaktion auf Gesells Pläne bekannt, da eine wissenschaftliche Untersuchung über die „Physiokraten“ oder, wie sie sich nach dem Ersten Weltkrieg nannten, über den „Freiland-Freigeld-Bund“ bzw. den „Freiwirtschaftsbund" bislang fehlt. ${ }^{75}$

Im Falle der DSP ${ }^{76}$ die 1920/21 als präfaschistische Vorläuferin der NSDAP in einigen Teilen Deutschlands eine gewisse Bedeutung erreichte, ist eine Beurteilung eher möglich. Hier sind viele Sitzungen belegt, auf denen vor allem um ein Wirtschaftsprogramm gerungen wurde, wobei sich zwei Gruppen jeweils um die beiden Hauptreferenten herausbildeten. Der eine war Gottfried Feder, der das 25-PunkteProgramm der NSDAP formulierte, und der andere war Silvio Gesell. ${ }^{77}$ Feder forderte den in Wirtschafts- und Währungsfragen souveränen Staat. Naiv glaubte er, daß der Staat zur Finanzierung nützlicher Projekte, z. B. von Wasserkraftwerken oder von Straßenbauten, jederzeit Geldschöpfung betreiben könne, da ja das fertiggestellte Werk ein Gegenwert für die neuen Zahlungsmittel sei und daher von Infla-

70 Walter Rathenau, Von kommenden Dingen, Berlin 1917. Gregor Straßer hat dieses Buch Rathenaus 1917 erworben und, wie seine persönliche Eintragung zeigt, mit im Felde gehabt. $\mathrm{Da}$ er es auch mit Interesse gelesen hat, bezeugen Randnotizen und viele Unterstreichungen, besonders auch in dem Kapitel über die Wirtschaft.

71 Schmid, a. a. O., S. 309.

72 Ebenda, S. $181 \mathrm{ff}$.

73 Ebenda, S. 258 ff.

74 Ebenda, S. 291.

75 Ebenda, S. 281, 293.

76 DSP = Deutsch-Sozialistische Partei (nicht zu verwechseln mit der DSP $=$ deutschsoziale Partei). Wolfgang Pfaar, Die Deutsch-Sozialistische Partei, Staatsarbeit, Düsseldorf 1974.

77 Mündl. Auskunft Pfaar. 
tion nicht die Rede sein werde. Daß solche Maßnahmen nur zur Ausnützung ungenutzter volkswirtschaftlicher Kapazität und gelegentlich zur Steuerung der Konjunktur getroffen werden dürfen, hat er nie erkannt. Er hat vielmehr das staatliche Recht auf Geldschöpfung als ein Recht sui generis betrachtet, das unabhängig von jeder Konjunkturlage bestehe und anwendbar sei. Anders als den komplizierten Konjunktursteuerungsmechanismus des Gesell'schen Freigeldes kann man die Thesen Feders nur als einen ,primitiven Inflationismus“ bezeichnen. ${ }^{78}$ Wichtig bleibt aber, daß in jenen frühfaschistischen Zirkeln der DSP diese Wirtschaftsprogramme diskutiert wurden. So fanden die Pläne Gesells zur Lenkung der Konjunktur Verbreitung, und durch Feder wurde hier wie dann vor allem in der NSDAP die grundsätzliche Möglichkeit der staatlichen Geldschöpfung von Anfang an gedankliches Allgemeingut. Unter den Personen, die in den Diskutiergruppen der DSP hervortraten, fallen in diesem Zusammenhang noch drei Namen auf, die später eine gewisse Bedeutung bekommen sollten: Dr. Herpel, ${ }^{79}$ Dickel ${ }^{80}$ und Dr. Bernhard Köhler ${ }^{81}$ Auch für Gregor Straßer ist bezeugt, daß er sich in den frühen zwanziger Jahren mit den Schriften Gesells beschäftigt hat, ${ }^{82}$ welche ihn sehr beeindruckten. Sein Bruder Otto hingegen stand als „ordentlicher“ Nationalökonom diesen neuen Lehren entsprechend seiner Ausbildung wesentlich kritischer gegenüber. Später ist es sogar zu einer persönlichen Aussprache zwischen den Brüdern Straßer und Silvio Gesell gekommen. ${ }^{83}$ Der ,Kampfverlag“, damals in Oranienburg beheimatet, lag in der Nachbarschaft von Gesells Wohnsitz in Eden bei Oranienburg, sodaß sich ein solches Treffen leicht hatte arrangieren lassen.

Bei der Neugründung der NSDAP im Jahre $1925^{84}$ gelangte ein weiterer GesellAnhänger in den Kreis der Mitglieder der Nordwestdeutschen AG, nämlich der Kreisleiter von Breslau und stellvertretende Gauleiter von Schlesien, Dr. Erich Rosikat. ${ }^{85}$ Er war eine der wichtigsten Personen der nationalrevolutionären Kreise Schlesiens und hatte dort als Syndikus der Landessiedlungsgesellschaft vor allem bei

78 Kroll, a. a. O., S. $244 \mathrm{ff}$.

79 Dr. Herpel, führendes Mitglied der DSP in Kiel; 1921 Kontakte zu Streicher (Angabe Pfaar), 1932 Mitarbeiter der ,Studiengesellschaft" (IfZ, ZS 248 Bd. III, S. 8), 1932 Mitarbeiter der „Querfront“ (IfZ, Akte Walter von Etzdorf, ZS 1926), 1932 Mitarbeiter Gerekes (Kurzorientierung des Ministeramtes 14. 7. 1932, IfZ, F 41).

80 Otto Dickel, Begründer der ,Werkgemeinschaft Augsburg“, 1922 wesentlich an der Auflösung der DSP beteiligt, bewegt auch Herpel zum Ausscheiden (Angabe Pfaar), 1932 Mitarbeiter der „Querfront“.

81 Dr. Bernhard Köhler, 2. Vorsitzender des „Kampfbundes zur Brechung der Zinsknechtschaft (Feder)“. Wesentlich an Wirtschaftsdiskussion der DSP beteiligt (Angabe Pfaar). Ab Mai 1932 Leiter der Unterabteilung Arbeitsbeschaffung der ROL (siehe auch Wagener-Tagebuch, S. $1343 \mathrm{ff}$., IfZ, ED 60).

82 Auskunft Otto Straßer v. 10.4.71.

83 Auskunft Otto Straßer v. 10.4. 1971. Dieser glaubt, daß es etwa 1928/29 gewesen sei. Genau erinnert er sich, daß es im Hause Gesells in Eden-Oranienburg stattgefunden hat.

${ }^{84}$ Siehe oben S. $28 \mathrm{ff}$.

${ }^{85} \mathrm{Zu}$ Erich Rosikat siehe Heiber, Goebbels-Tagebuch, S. 29, 78f., 84, 106ff., Tyrell, a. a. O., S. 122, 144, $166 \mathrm{ff} ., 280$. 
der ländlichen Bevölkerung einen starken Rückhalt. Sowohl von den Bodenreformern als auch vor allem von Gesell beeinflußt, widmete er sich diesem kleinbürgerlichen Siedlungsprogramm, das auf dem Boden des Großgrundbesitzes durchgeführt werden sollte, mit ganzer Kraft. Seine Pläne und Vorstellungen vertrat er auch in vielen Veröffentlichungen. Nachdem ihn seine Aktivitäten zunächst in die Nähe der schlesischen DSP ${ }^{86}$ geführt hatten, brachte er 1925 seine schlesischen Anhänger in die neue NSDAP ein, in der er sich wohl nur deshalb mit dem stellvertretenden Gauleiterposten unter Brückner begnügte, weil er sich als Schwerkriegsversehrter aus gesundheitlichen Gründen kaum mehr zumuten konnte. 1927 wurde er als erster Prominenter der AG von Hitler aus der Partei ausgeschlossen. Danach schloß er sich lose der Ludendorff-Bewegung an, bis er 1934 nach schwerer Krankheit starb.

Von seinen Veröffentlichungen, die vor allem in völkischen Zeitungen Schlesiens erschienen, sind nur noch wenige erreichbar, ${ }^{87}$ bei denen es sich leider nicht um programmatische Aufsätze handelt, sondern um Stellungnahmen zu aktuellen Fragen der Siedlung. Doch sind auch aus ihnen in Umrissen die „Freiland“-Ideen Gesells zu erkennen. Rosikat forderte die Ansiedlung von Deutschen auf Kleinbauernstellen, die durch Verstaatlichung des Großgrundbesitzes gegen Entschädigung zu schaffen seien. Er dachte dabei vor allem an die Sicherheit des Deutschtums im Osten. Daneben aber - und hier wird die Nähe zu Gesell deutlich - wollte er die allgemeine Arbeitslosigkeit bekämpfen und die Wirtschaftskonjunktur positiv beeinflussen. So schrieb er: „Die Vermehrung des seßhaften Landvolkes ist daher nicht nur mit Rücksicht auf die Polengefahr, sondern auch im Hinblick auf die brennende Erwerbslosenfrage von höchster Bedeutung. Gelingt es, den Landmann von der Zuwanderung zur Stadt abzuhalten, so wird der Erwerbslosenmarkt entlastet. Jede neue Bauernstelle bedeutet eine Entlastung in diesem Sinne. Man muß berücksichtigen, daß durch den Kleinbetrieb die Landbevölkerung gegenüber dem Großbetrieb auf der gleichen Fläche um das Dreifache an Zahl gesteigert werden kann. Daneben aber bietet die Durchführung der Siedlung selbst umfangreiche Arbeitsgelegenheit. In jeder neuen Siedlung stecken an Arbeitsleistung im Durchschnitt unmittelbar 1600 Tagewerke, dazu kommt die Belebung der Industrien, welche Baustoffe, Ackergeräte usw. herstellen. Mit den Siedlerfamilien finden Kaufleute, Handwerker usw. eine neue Existenz. Landeskulturarbeiten werden gefördert: Entwässerungen, Wegebauten, Obstplantagen, Schulbauten entstehen.“ “88 Zur Finanzierung solcher Maßnahmen schlug er ein System von Staatskrediten und Beihilfen vor, wobei er insbesondere auf die Möglichkeit von Anleihen und auf die Voranrechnung von späteren Steuern verwies. ${ }^{89}$ Zusammen bedeutete das die, wenn auch noch unklar ausgesprochene, Forderung nach staatlicher Geldschöpfung.

${ }^{86}$ DSP (deutsch-soziale-Partei) unter Kunze. Auskunft Hans Kurth v. 7. 10. 1971.

E. Rosikat, Die Finanzierung der landwirtschaftlichen Siedlung, in: Archiv für innere Kolonisation, Bd. 16 (1924) Heft 4/7, A. 125-136; ders., Die Siedlung als Landvolkbewegung, in: Volk und Reich, Berlin 1926, Bd. II, S. 540-545.

88 E. Rosikat, Die Finanzierung S. 132.

89 Vgl. die späteren Steuergutscheine der Regierung v. Papen. 
1925/26 nahm Rosikat an den Hannover-Tagungen ${ }^{90}$ teil und erhielt dadurch Einfluß auf Gregor Straßers „Bamberger-Programm“.91 Daneben wurde Gregor vor allem von seinem Bruder Otto beraten. Otto Straßer hatte als Nationalökonom 1921 über das landwirtschaftliche Thema „Entwicklung und Bedeutung der deutschen Zuckerrübensamenzucht" in Würzburg promoviert ${ }^{92}$ und danach als Hilfsreferent im Reichsernährungsministerium unter Minister Hermes (Zentrum) gearbeitet; er hatte ein Sonderreferat über landwirtschaftliche Meliorationen geleitet ${ }^{93}$ Nach einigen Jahren Tätigkeit in der Industrie war er 1925 zur Unterstützung seines Bruders der NSDAP beigetreten.

Da Rosikat und Otto Straßer, die einzigen Mitglieder der AG, die einige praktische wirtschaftspolitische Erfahrung besaßen, beide Landwirtschaftsfachleute waren, ist es nicht verwunderlich, daß der entstehende Programmentwurf auf agrarpolitischem Gebiet überbetont war, worin natürlich auch der rückwärts gerichtete und an vorindustriellen Strukturen orientierte Charakter der gesamten völkischen Bewegung zum Ausdruck kam. Im Bamberger Programm, einem der wichtigsten Frühzeugnisse der NSDAP, namentlich hinsichtlich der Punkte, die sich mit Wirtschaftspolitik beschäftigen, sind aber neben der auffallenden Bevorzugung der Agrar- vor der Industrie-Wirtschaft Forderungen zu finden, die eindeutig auf eine staatliche Lenkung des Wirtschaftssystems zielen und auch die Gesell'sche Hauptforderung nach einer Boden- und Währungsreform aufgreifen. Ähnlichkeiten mit Peter Straßers Aufsatz aus dem Jahre 1912 sind da, wo es um die Verstaatlichung des Bodens und der Industrie geht, ebenfalls zu erkennen. Wie Otto Straßer berichtet, war dieser Aufsatz den Teilnehmern der Hannover-Konferenz tatsächlich in einigen Exemplaren zugänglich gemacht worden. ${ }^{94}$ So verlangte das Programm ${ }^{95}$ Siedlungsprojekte auf dem Grund und Boden, der Eigentum der Nation sei, und zwar die Schaffung sowohl bäuerlicher Güter als auch von Nebenerwerbsstellen für landwirtschaftliche Arbeiter. Zugleich müsse, so hieß es, eine umfassende Flurbereinigung durchgeführt werden. Die Bauern seien zu Genossenschaften zusammenzuschließen, während die Industrie, nach einem komplizierten Schlüssel, weitgehend in den ,Besitz der Allgemeinheit“" überführt und dadurch auch lenkbar gemacht werden sollte. „Beständige Úberwachung der Modernität des technischen Standes, eventuell unter Stillegung unrentabler Werke, ist ebenfalls Sache des Reichswirtschaftsministeriums." Das Bamberger Programm nahm sich auch schon der Währungsfrage an. So wurde eine Spaltung der Währung in Inlands- und Auslandswährung gefordert, wobei es von der ersteren hieß: „Inlandswährung gleich Arbeitsmarkt“. Hier wurde, wenn auch nur in Schlagwortform, die Idee des volkswirtschaftlichen Kreditfonds vorwegge-

90 Auskunft Otto Straßer v. 10.4. 1971.

91 Kühnl, Das Straßer-Programm von 1925/26, S. 317, Kühnl, Die nationalsozialistische Linke, S. $20 \mathrm{ff}$.

92 Otto Straßer, Mein Kampf, S. 20.

93 Otto Straßer, Mein Kampf, S. 20f. Auskunft Otto Straßer v. 10.4. 1971.

94 Auskunft Otto Straßer v. 10. 4. 1971.

95 Otto Straßer, Mein Kampf, S. 20. 
nommen. ${ }^{96}$ Die Auslandswährung sollte im Gegensatz dazu ans Gold gebunden bleiben, um den notwendigen Außenhandel aufrecht zu erhalten. Zusätzlich war allerdings auch an einen mitteleuropäischen Zollverein oder gar an die Vereinigten Staaten von Europa gedacht.

Wenngleich dieser Programmentwurf und damit die AG in Bamberg 1926 noch scheiterten, gab Gregor Straßer weder die Ziele der AG noch den Inhalt seines Programms endgültig verloren. ${ }^{97}$ Besonders die wirtschaftspolitischen Forderungen verfolgte er weiter. Stand er 1926 noch im Schatten des Fachwissens seines Bruders und unter dem Einfluß Rosikats, so hatten sich diese Verbindungen gelöst, als er sich einige Jahre später, unter dem Druck der beginnenden Wirtschaftskrise, wieder eingehender zu solchen Fragen äußerte. War das Bamberger Programm noch Ergebnis einer Diskussion gleichberechtigter Mitglieder der „Nordwestdeutschen AG“ gewesen, so stützte sich Straßer inzwischen mehr und mehr auf die Arbeiten der Fachleute in der ihm unterstehenden ROL. Obgleich sich die personelle Situation in seinem Stab nur langsam besserte, versetzten ihn seine - noch immer zu wenigen Experten jetzt doch in die Lage, zu Wirtschafts- und Konjunkturfragen fundierter Stellung zu nehmen und allmählich ein Wirtschaftsprogramm für die NSDAP ausarbeiten zu lassen. Unmittelbar nach dem Wahlerfolg vom 14. September 1930 ergriff er am 17. Oktober $1930 \mathrm{im}$ Reichstag das Wort zu einer grundsätzlichen Erklärung im Namen der NS-Fraktion. In demagogischer Form ging er das Problem der Arbeitslosigkeit an, attackierte er die Finanz- und Wirtschaftspolitik der Regierung. In seinem Ruf nach einer neuen Wirtschaftsordnung waren verschwommen die Ideen von 1926 und auch die von Gesell wieder zu erkennen. ${ }^{98}$ „Unser nächstes Ziel ist daher die Beseitigung des Leerlaufs der deutschen Wirtschaft, und zwar erstens durch Úberwindung der Arbeitslosigkeit und Gesundung der Landwirtschaft, zweitens durch Lösung der Frage des gerechten Arbeitslohnes und drittens durch Neuordnung unserer Geldwirtschaft.“" Er behauptete, daß die „Uberproduktion“" ein Schwindel sei. In Wahrheit sei das Wirtschaftssystem nicht in der Lage, „Produzenten und Konsumenten" zusammenzubringen. Besonders trostlos sei die Situation der Landwirtschaft: „Dem Landbau fehlt die gesamte ökonomische, soziale Grundlage. Weite Stätten liegen brach, und selbst bebautes Land verödet, weil es an Arbeitskräften fehlt.“ Zum Währungssystem bemerkte er: „Wir verlangen ein ehrliches Währungssystem, ein Währungssystem, das sich von dem Goldwahn der heutigen Zeit freimacht, ein System, das das Problem der Inlandwährung, auf Sachwerten gedeckt, durchstudiert und erfüllt." Ferner verlangte er ein autarkes Wirtschaftssystem und „Arbeitsbeschaffung" durch staatliche Baumaßnahmen.

Straßers wirtschaftspolitische Forderungen waren freilich noch dilettantisch vorgetragen, verschwommen formuliert und keineswegs schon zu einem einheitlichen Konzept verbunden. Da er selbst weder Fachwissen noch die Zeit hatte, sich gründli-

\footnotetext{
96 Kroll, a. a. O., S. 443 f.

97 Siehe oben S. $26 \mathrm{f}$.

98 Verhandlungen des Reichstages, Bd. 444, S. $60 \mathrm{a}$, b.
} 
cher in die Materie einzuarbeiten, wurde es immer notwendiger für ihn, geeignete Mitarbeiter und Berater für seinen Wirtschaftsstab zu finden, und zwar solche, die als Vertreter der NSDAP in einem NS-Staate die Regierungs- und Staatsgeschäfte übernehmen konnten; er war ständig auf der Suche nach solchen Fachleuten. ${ }^{99}$ Typisch für ihn war, daß er dabei, wie schon das Beispiel seiner Kontaktaufnahme mit Oswald Spengler im Jahre 1926 gezeigt hatte, durchaus bereit war, falls sich innerhalb der Partei kein geeigneter Mitarbeiter fand, mit Personen außerhalb der Partei zusammenzuarbeiten, sofern sie zu den im nationalen Sinne ,aufbauwilligen Kräften" gehörten. ${ }^{100}$ Zwar hatte Feder, der offizielle Parteiprogrammatiker, eigene wirtschaftspolitische Vorstellungen entwickelt, doch waren sie nicht über das unklare Geldschöpfungssystem hinausgekommen, das er schon Anfang der zwanziger Jahre in jenen Diskussionen bei der DSP vorgetragen hatte. ${ }^{101}$ Damit konnte Straßer offensichtlich nicht viel anfangen. Er sah überhaupt keinen brauchbaren Wirtschaftsfachmann in der Partei. ${ }^{102}$ So baute er seit Anfang 1931, verstärkt seit Winter 1931/32, die wirtschaftspolitische Abteilung unter Otto Wagener aus. ${ }^{103}$ Nun schlossen sich gerade 1931, als sich die verschiedenen Silvio-Gesell-Gruppen und -Vereine auflösten, deren Anhänger scharenweise der NSDAP an. Die „Freigeld“Theorien Gesells wurden daher in zunehmendem Maße auf allen Ebenen der Partei diskutiert. Bei einigen älteren Parteimitgliedern löste das Besorgnis aus, bereitete aber andererseits den innerparteilichen Boden für ein neues Wirtschaftsprogramm Straßers. ${ }^{104}$ Die Aufgabe der wirtschaftspolitischen Abteilung sollte im wesentlichen in der Ausarbeitung eines Wirtschaftsprogramms für eine spätere Regierungsübernahme bestehen. Den Angehörigen der Abteilung wurde offensichtlich die Weisung gegeben, sich auch mit Gedanken außerhalb der Partei vertraut zu machen und sich in geeigneter Weise allenthalben an der Erarbeitung neuer Programme zu beteiligen. Tatsächlich kamen denn auch von außerhalb der Partei stehenden Kreisen entscheidende Anregungen, mit denen Straßer seine eigenen wirtschaftspolitischen Vorstellungen zu einem Programm konkretisierte.

\section{Die „Reformer“"}

Die Ideen zur Beschreitung neuer Wege, wie sie zu Beginn der Weltwirtschaftskrise, angeregt durch die Lehre Gesells, gerade in Deutschland entstanden, ${ }^{105}$ bewegten sich weitgehend außerhalb der klassischen liberalen Wirtschaftstheorie und zielten

\footnotetext{
99 Wagener-Tagebuch, S. $674 \mathrm{ff}$.

100 Siehe oben S. 19.

101 Siehe oben S. 96.

102 Wagener-Tagebuch, S. 661.

103 Tyrell, a. a. O., S. $362 \mathrm{f}$.

104 Brief Dr. Albrecht an Oberst Hierl vom 12. 1.1932 und Entwurf einer Anordnung vom 3. 2. 1932 , BA, NS $22 / 11$.

105 Jörg Ohl, a. a. O., S. 2: „,Die deutsche Theorie hat durchaus nicht erst mit dem Erscheinen
} 
nicht auf eine grundsätzliche, theoretische Lösung der Konjunkturfrage, sondern nur auf praktische Ergebnisse. So erklärte Professor Wagemann 1932 öffentlich, daß alle Diskussionen nur der praktischen Úberwindung der Krise zu dienen hätten, wozu theoretische Klarheit über die Ursachen der Krise nicht notwendig sei. ${ }^{106}$ Neben dem Anstoß von Silvio Gesell waren es Tatsachen, die zum Denken anregten: Zum einen die Beobachtung, daß in den zwanziger Jahren eine relativ hohe Grundarbeitslosigkeit bestand; ${ }^{107}$ auch in den Jahren des höchsten Aufschwungs, 1927 und 1928, sanken die Arbeitslosenziffern im Jahresdurchschnitt nicht unter 1,391 Millionen, eine Höhe, die man in den Jahren vor dem Ersten Weltkrieg nicht gekannt hatte. ${ }^{108}$ Zum anderen war es die Erinnerung an die Staatskonjunktur in Deutschland während des Ersten Weltkrieges, die unter Lösung vom Goldstandard zu ganz erstaunlichen Wirtschaftsleistungen fähig gewesen war. Die Bedeutung dieser Erinnerung für die mit der Weltwirtschaftskrise einsetzende Meinungsbildung in der interessierten Öffentlichkeit Deutschlands ist mit folgenden Sätzen zutreffend charakterisiert worden: „Mehr als in jedem anderen kapitalistischen Land war in Deutschland die Bereitschaft vorhanden, die Selbststeuerung des Systems durch eine das gesamte Wirtschaftsleben umspannende, zentralistisch geleitete Planwirtschaft zu ersetzen. Das Vorbild war die „Kriegsstoff-Abteilung“ Rathenaus während des Weltkrieges, die als der erste funktionierende Apparat einer Planwirtschaft angesehen werden kann." ${ }^{109}$ Der wichtigste Autor für die in Deutschland mit Beginn der Weltwirtschaftskrise einsetzende Diskussion über Wirtschafts- und Konjunkturfragen, Robert Friedländer-Prechtt ${ }^{110}$ - Silvio Gesell war am 11. März 1930 gestorben - hatte bereits 1926 auf jene beiden Tatsachen in seinem Buche „Chronische Arbeitskrise, ihre Ursachen, ihre Bekämpfung " ${ }^{111}$ hingewiesen. Am Schluß seiner Untersuchung hatte er die Forderung nach Überwindung der Arbeitslosenkrise nachdrücklich betont: „Die disponiblen Arbeitskräfte und disponiblen Arbeitsmittel - beide zusammen stellen ein ungeheures brachliegendes Kapital dar - müssen zusammengebracht, die entstehenden Produkte zu Investierungszwecken dem

der General Theory ... ihr Augenmerk dem Beschäftigungsproblem zugewandt. Sie hat vielmehr ... fast als einzige außerhalb der angelsächsischen Welt entscheidend an der Entwicklung der neuen Gedanken mitgearbeitet und ist dabei in durchaus selbständiger Weise zu allen wesentlichen Erkenntnissen der modernen Lehre gelangt.“

106 Prof. Wagemann am 3. August 1932, zitiert nach Grotkopp, a. a. O., S. 43. Vgl. auch Ohl, a. a. O., S. 8: „Es war weniger die Frage nach der theoretischen Ursachenlage, als die Frage der praktischen Beseitigung der Arbeitslosigkeit."

107 Robert Friedländer-Prechtl, Chronische Arbeitskrise, ihre Ursachen, ihre Bekämpfung, Berlin 1926, S. 6.

108 Gerhard Bry, Wages in Germany 1871-1945, Ann Arbor Michigan 1967, S. 156 ff. u. $297 \mathrm{f}$., Tafel A $1+28$.

109 Jörg Ohl, a. a. O., S. 6.

110 Robert Friedländer-Prechtl, geb. 1881, Studium der Nationalökonomie und der Rechtswissenschaften, Teilhaber des Kohlekonzerns Friedländer-Fuld, als Finanzier beteiligt an der Entwicklung des Bergius-Kohleverflüssigungsverfahrens und an den ersten Fabriken zur Kunstharz- und Kunstseideherstellung.

111 Robert Friedländer-Prechtl, Chronische Arbeitskrise, S. 6, 34. 
In- und Auslande zugeführt werden. Als Gegenwert kommen zunächst langfristige Anleihen und Schuldtitel herein, die gegebenenfalls durch Passivanleihen des Reiches mobilisiert werden, ... Die Mittel für solche Wirtschaftspolitik sind vorhanden, denn Arbeitskräfte und Arbeitsmittel, die einzig realen Werte, sind in ausreichendem Maße da. Sie zusammenzubringen und ihren Zwecken zuzuführen, ist allein eine finanztechnische Frage, die gelöst werden wird - weil sie gelöst werden mиß! “112

In einer zweiten Veröffentlichung, die unter dem Titel „Die Wirtschaftswende“ 1931 erschien $^{113}$ und ganz unter dem Eindruck der großen Krise stand, präzisierte er seine Ideen. Abermals war für ihn die Zeit des Ersten Weltkrieges das augenscheinlich positive Beispiel einer Staatskonjunktur, im Gegensatz zur „Laissez-faire-Politik“ des reinen Wirtschaftsliberalismus: „Deutschland hatte während der Kriegsjahre gewiß kein Geld und keinen Kredit, beides friedenswirtschaftlich betrachtet. Und gleichwohl hat es in dieser Zeit Anlagen größten Umfangs geschaffen: Bahnen und Brücken, Maschinenwerkstätten und Chemiefabriken, davon zum Teil solche, die - wie die Stickstoffwerke - auch für Friedenszwecke Dauerwerte darstellten. Es wurde ein Wirtschaftsplan aufgestellt, das „Hindenburg-Programm“, und nach diesem schuf die Wirtschaft alles das, was für die Bedürfnisse des Krieges erforderlich war, soweit hierzu die im Inland vorhandenen Rohstoffe ausreichten."114

Einen weiteren wichtigen Anstoß auf dem Wege zu neuen Ideen gab das Gutachten einer von Brüning eingesetzten Kommission unter Leitung des früheren Arbeitsministers Braun, kurz „Brauns-Gutachten“ genannt. ${ }^{115}$ Es wurde im April und Mai des Jahres 1931 in zwei Teilen veröffentlicht. Während im ersten Teil nur traditionelle Vorschläge wie Arbeitszeitverkürzung ohne Lohnausgleich und Abschaffung von Doppelverdienertum wiederholt wurden, klangen im zweiten Teil neue Ideen an. Die Kommission zählte eine Fülle von öffentlichen Projekten zur Arbeitsbeschaffung auf und behandelte auch das Problem der Finanzierung solcher Maßnahmen. Sie glaubte, daß etwa 2 Milliarden RM notwendig seien und daß diese Summe vom Staat als Auslandsanleihe aufgenommen werden sollte. Von der bahnbrechenden Erkenntnis abgesehen, daß man zur Steuerung der Krise aktive Konjunkturpolitik in Form von staatlicher Arbeitsbeschaffung betreiben könne, blieb sie also hinsichtlich der Finanzierung noch in alten Vorstellungen verhaftet. Es muß allerdings darauf hingewiesen werden, daß die Kommission auch hier bereits Neuland zu betreten versuchte. Sie erklärte nämlich in einer Nebenbemerkung, daß im Zusammenhang mit einer Auslandsanleihe durchaus auch kleinere Rückgriffe auf die Reichsbank in Aussicht genommen werden könnten. ${ }^{116}$ Freilich hatte das „Brauns-Gutachten“ keinen direkten politischen Einfluß. Jedoch lieferte es der Ministerialbürokratie die

112 Ebenda, S. 167.

113 Robert Friedländer-Prechtl, Die Wirtschaftswende, Leipzig 1931.

114 Ebenda, S. 229 ff.

115 Brauns-Gutachten, Kroll, a. a. O., S. 377.

116 Ebenda, S. 379. 
Grundlage für weitere Pläne, ${ }^{117}$ die es dann vor allem der Regierung Hitler ermöglichten, Wirtschaftsankurbelungen schneller vorzunehmen, da wichtige Vorarbeiten schon geleistet waren. Auch regten die Pläne der Kommission die Diskussion über eine neue Wirtschaftspolitik nachhaltig an. Einen weiteren Anstoß gab eine Maßnahme der Regierung Brüning. Als am 13. Juli 1931 deutsche Banken in Schwierigkeiten kamen, ausgelöst durch einen Run der Konteninhaber auf die Kreditinstitute nach dem Zusammenbruch der „Österreichischen Kreditanstalt", reagierte Brüning schnell. Im Gegensatz zur liberalen Wirtschaftstheorie von den Selbstheilungskräften griff er hart durch. Eine Notverordnung schloß die Schalter aller Bank- und Sparinstitute für den 14. und 15. Juli.

Die Regierung übernahm die Garantie für die Einlagen bei der Danatbank und später auch bei anderen Banken. Nach der Wiedereröffnung der Schalter wurden, ebenfalls durch eine Notverordnung, sowohl die Höhe von Abhebungen und Überweisungen festgelegt als auch Maßnahmen gegen die Kapital- und Steuerflucht ergriffen. Im Laufe des August stabilisierte sich das Bankensystem wieder, so daß man auch schrittweise die Einschränkungen von Abhebung und Uberweisung aufheben konnte. Brüning hatte mit Erfolg ,aktive“ Wirtschaftspolitik betrieben, allerdings nur in einem ,passiven“ Sinne, um Schlimmeres zu verhüten, und nicht etwa, um die Konjunktur wieder aufzubauen. Für die zeitgenössische Wirtschaftsdiskussion allerdings wurde diese Aktion zu einem immer wieder zitierten Paradefall. ${ }^{118}$ Freilich wußten, trotz zunehmender Verschärfung der Wirtschaftskrise, weder die Politiker noch die professionellen Nationalökonomen einen besseren Rat, als abzuwarten, bis die Krise sich selbst behebe. Es waren vor allem sogenannte „Nichtfachleute“ und Außenseiter, die eine grundsätzliche Reform der Wirtschafts- und Konjunkturpolitik öffentlich forderten. Aus der Vielzahl dieser „Reformer", wie sie sich selbst gerne nannten, ${ }^{119}$ ragten einige Gruppen besonders hervor. Der bereits erwähnte Robert Friedländer-Prechtl gründete im Jahre 1931, in dem er auch sein gleichnamiges Buch veröffentlichte, die in München erscheinende Zeitschrift „,Die Wirtschaftswende", um die ein Kreis Gleichgesinnter entstand. Professor Wagemann, ${ }^{120}$ der Leiter des statistischen Reichsamtes und des Instituts für Konjunkturforschung, beschäftigte sich ebenfalls, zusammen mit seinen Mitarbeitern, mit neuen Ideen, die er Anfang 1932 im sogenannten Wagemann-Plan vorlegte. In Nürnberg sammelte sich eine Gruppe um Professor Vershofen und den jungen Nationalökonomen Ludwig Erhard. ${ }^{121} \mathrm{Im}$ „Tatkreis“ entwickelte Ferdinand Friedrich Zimmermann (Ferdinand

117 Auskunft Schwerin-Krosigk v. 28. 11. 1971.

118 Kroll, a. a. O., S. 122, S. 362, Grotkopp, a. a. O., S. 16f.; Verhandlungen des Reichstages, Bd. 446, S. 2514 c.

119 Grotkopp, a. a. O., S. 8 f.

120 Kroll, a. a. O., S. $396 \mathrm{ff}$.

121 Prof. Wilhelm Vershofen, Ordinarius an der Handelshochschule Nürnberg, Leiter des Instituts für Wirtschaftsbeobachtung der deutschen Fertigware. Dr. Ludwig Erhard war damals Dozent am gleichen Institut, dessen Leitung er 1934 übernahm. 
Fried) neue Gedanken. ${ }^{122}$ Große Bedeutung erlangte ferner der Wirtschaftsreformerkreis im ADGB um Woytinski, Tarnow und Baade. ${ }^{123} \mathrm{Zu}$ diesem Kreis darf wohl auch Erdmann, der Chefredakteur der Gewerkschaftszeitung „Die Arbeit" gerechnet werden, der in seinem Blatt vor allem Woytinski Platz zur Verbreitung seiner Ideen einräumte. ${ }^{124}$ Zuletzt ist noch der „Gereke-Kreis“ zu nennen, auf den später näher eingegangen werden muß. Um Gereke, Präsident des Landgemeindetages und Reichstagsabgeordneter, bildete sich eine Reformer-Gruppe aus den verschiedensten politischen Lagern. Zunächst nur an rein wirtschaftsreformerischen Fragen interessiert, wurde die Gruppe im Laufe des Jahres 1932 aber auch zu einer wichtigen innenpolitischen Schaltstelle. Neben Gereke selbst spielten in ihr vor allem der Industrielle und Stahlhelmführer Lübbert und Walther von Etzdorf eine Rolle. ${ }^{125}$ Die Grenzen zwischen all diesen Gruppen waren fließend. Es gab zahlreiche Querverbindungen.

Angeregt durch die erwähnten Bücher von Friedländer-Precht1 ${ }^{126}$ und besonders auch durch seine Zeitschrift, war es schließlich ein dort im Herbst 1931 publizierter Artikel von Dalberg ${ }^{127}$ „Wirtschaftslähmung durch Prinzipien“, ${ }^{128}$ der den Industriellen Heinrich Dräger ${ }^{129}$ dazu bewog, den Verfasser Dalberg persönlich aufzusuchen. In einer Unterredung, in der sich beide schnell grundsätzlich einigten, faßten sie den Entschluß, gemeinsam eine Plattform für alle zu schaffen, die an einer neuartigen aktiven Wirtschaftspolitik interessiert waren. ${ }^{130}$ Etwa im November 1931 entstand hieraus in Berlin „Die Studiengesellschaft für Geld- und Kreditwesen“. In ihrer ersten Veröffentlichung bezeichnet sie sich selbst als ,eine zwanglose Vereinigung mit dem Ziel, für die Erörterung der Probleme der Geld- und Kreditwirtschaft, die heute wieder so sehr im Vordergrund des allgemeinen Interesses stehen, einen neutralen Rahmen zu schaffen. Die Studiengesellschaft beabsichtigt, zu diesem Zweck Vorträge und Diskussionsabende zu veranstalten und gegebenenfalls auch einschlägige Veröffentlichungen zu fördern. Die Studiengesellschaft ist parteipoli-

122 Die Tat, Heft 6, September 1932; Ferdinand Fried, Der Umbau der Wirtschaft, S. 452 ff.; Grotkopp, a. a. O., S. 39, 42.

123 WTB-Plan der Gewerkschaften, siehe unten S. 108.

123 Grotkopp, a. a. O., S. 41.

125 Günther Gereke, Ich war königlich-preußischer Landrat, Berlin 1970, S. $195 \mathrm{ff}$.

126 Siehe Anm. IV 110, 112.

127 Rudolf Dalberg, geb. 1885; 1919 Regierungsrat im Reichsfinanzministerium, 1923 im Reichswirtschaftsministerium Hauptreferent für Geld- und Bankwesen, Mitwirkung bei der Schaffung der neuen Währung, 1926 Leitung der deutschen Abteilung der BritishGerman-Trust Ldt., London, mit Sitz in Berlin.

128 Entwurf einer Einleitung von Dr. H. Dräger, IfZ, ZS 248, Bd. III S. 6.

129 Dr. Heinrich Dräger, geb. 1898, seit 1928 Fabrikant in Lübeck.

130 Dräger, Entwurf, S. 6 ff.; Grotkopp, a. a. O., S. 35f.; Kroll, a. a. O., S. 404. Vgl. auch Henning Köhler, Arbeitsbeschaffung, Siedlung und Reparationen in der Schlußphase der Regierung Brüning, VfZ 17 (1969), S. 276-307, ferner Horst Sanmann, Daten und Alternativen der deutschen Wirtschafts- und Finanzpolitik in der Ära Brüning, in: Hamburger Jahrbuch für Wirtschafts- und Gesellschaftspolitik Bd. 10 (1965), S. 109-140. 
tisch neutral und nimmt als solche an sich keine Stellung zu irgendwelchen Fragen oder Vorschlägen. Ihre Veranstaltungen sind jedoch von der Überzeugung getragen, daß die gegenwärtigen geld- und kreditwirtschaftlichen Verhältnisse nicht mehr den an sie zu stellenden Anforderungen genügen, daß die gegenwärtige Wirtschaftsdepression durch Maßnahmen auf dem Gebiet der Geld- und Kreditpolitik gemildert oder überwunden werden kann." 131 Als wichtigen Mitarbeiter konnten Dräger und Dalberg bald Professor Wagemann gewinnen. Als anerkannter Nationalökonom brachte er, außer seinem Fachwissen und den speziellen Untersuchungen seines Mitarbeiterstabes, der „Studiengesellschaft“ ein gewisses Maß an fachlicher Seriosität, das ihr Ansehen und ihre Einflußmöglichkeiten wesentlich verstärkte. ${ }^{132} \mathrm{Im}$ engsten Kreise wirkten außerdem Grotkopp ${ }^{133}$ als Schriftführer und Grävell ${ }^{134}$ mit. Vor allem durch Vorträge und als Diskussionsredner beteiligten sich: Wildermuth, Sombart, Otto Christian Fischer, Gereke, Otto Hahn, Lautenbach, Gestrich, Woytinski, Friedländer-Prechtl, Lampe, Prion und der schon bei den DSP-Diskussionen erwähnte Dr. Herpel. Auch die NSDAP war vertreten, und zwar in erster Linie durch Mitarbeiter aus Straßers Reichsorganisationsleitung. ${ }^{135} \mathrm{Zu}$ den Mitbegründern der Studiengesellschaft gehörte der Dozent der Nationalökonomie Jens Jessen, der dem Gauleiter Rust nahestand und damals aktiver Nationalsozialist war, wenn auch seine formale NS-Mitgliedschaft später in Zweifel gezogen wurde. ${ }^{136}$ Daneben beteiligten sich Werner Daitz ${ }^{137}$ und Fritz Reinhardt, ${ }^{138}$ beide damals Mitglieder des Wirtschaftsrates der ROL der NSDAP, Reinhardt gleichzeitig Reichspropagandaleiter II und Gauleiter von Oberbayern. Schließlich wirkten noch Hermann Tholens, Leiter der Unterabteilung Arbeitsbeschaffung und ab 10. Juni 1932 Leiter der Abteilung „Öffentliche Siedlung“ in der Reichsorganisationsleitung der NSDAP, sowie Arthur R. Herrmann, Leiter der Abteilung Währung und Geldwesen der ROL, mit. ${ }^{139}$ Úber die Berliner Börsenzeitung bestanden persönliche Verbindungen von

131 Grotkopp, a. a. O., S. 40.

132 Schäffer Tagebuch, IfZ, ED 93, Bd. 17, S. 126, 135.

133 Wilhelm Grotkopp, geb. 1900; Auslandskorrespondent und Wirtschaftspublizist beim Ullstein-Verlag, ab 1930 Herausgeber der ,Europa-Wirtschaft".

134 Walter Grävell, geb. 1891; nach dem Weltkrieg Abteilungsleiter im statistischen Hauptamt, unter Luther in der Reichskanzlei, später Leiter des Enquete-Ausschusses, auch freier Mitarbeiter an der ,Europa-Wirtschaft".

135 Vgl. Grotkopp, a. a. O., S. 37, Dräger, a. a. O., S. 44, 75, 79, 81, 88, 91.

136 Grotkopp, a. a. O., S. 37, 39 und 39 Anm. 3. Jens Jessen, geb. 1896; habilitierte sich 1927 in Göttingen, 1933 Ordinarius in Kiel und Direktor des Weltwirtschaftsinstitutes, am 30. 11. 1944 als Beteiligter am 20. Juli 1944 hingerichtet.

137 Werner Daitz, geb. 1884; Industrieller aus Lübeck, Mitglied des Herrenclubs, Mitglied des Wirtschaftsrats der ROL, BA, NS 26/1283.

138 Reinhardt war damals Leiter der Reichspropagandaleitung II. Ab 30. 1. 1933 Staatssekretär im Reichsfinanzministerium. Nach ihm wurde der Arbeitsbeschaffungsplan der Regierung Hitler „Reinhardt-Plan“ genannt.

139 Grotkopp, a. a. O., S. 37. 
Dalberg zu Funk, ${ }^{140}$ der dadurch in Kontakt zu den Reformern kam, was wohl auch zu Treffen von Funk und Wagemann geführt hat, ${ }^{141}$ obgleich man Funk sicherlich nicht zum eigentlichen Kreis der Reformer rechnen darf. ${ }^{142}$ Selbst mit Keynes nahmen die Reformer Verbindung auf, als dieser zu einem Vortrag in Hamburg weilte. Allerdings zog Keynes seine dort gemachte Zusage zu einem Vortrag in Berlin auf eine Intervention der Reichsbankleitung hin zurück. ${ }^{143}$ Im übrigen geht aus der Liste der Vortragenden hervor, daß auch Fäden zu Gewerkschaftlern geknüpft worden waren; vor allem Dräger unterhielt sowohl zu Woytinski als auch zu Tarnow enge Beziehungen. Mit einer dritten Gruppe, die im Laufe des Jahres 1932 auf sich aufmerksam machte, dem sogenannten „Gereke-Kreis“, wurde ebenfalls eng zusammengearbeitet. In diesem Zusammenhang ist besonders bemerkenswert, daß Gereke, damals Präsident des deutschen Landgemeindetages und Reichstagsabgeordneter der Landvolkpartei, neben guten Beziehungen zur Reichswehr, d.h. vor allem zu Schleicher, und zur gemäßigten nationalen Rechten vor allem auch enge Kontakte zur SPD und zum ADGB besaß. ${ }^{144}$ Alle drei Gruppen, die Studiengesellschaft, die Gewerkschaftler unter Führung von Woytinski bezw. Tarnow und Baade sowie der Gereke-Kreis, standen in dauernder Verbindung. Straßer war über diese Aktivitäten mit Sicherheit ausgezeichnet unterrichtet und verfolgte sie wohlwollend; jedenfalls hatte man in der Studiengesellschaft diesen Eindruck, ${ }^{145}$ wenngleich er sich zunächst nicht persönlich beteiligte, sondern sich durch seine Mitarbeiter vertreten ließ. Indes kam es wahrscheinlich schon im Herbst 1931 zu einem Treffen Gerekes mit Gregor Straßer im Reichstag, wobei beide über die Notwendigkeit eines staatlichen Arbeitsbeschaffungsdienstes im Grundsatz Einigkeit erzielten. Hieraus entwickelte sich ein ständiger Kontakt, der allerdings nicht von Straßer persönlich wahrgenommen, sondern von seinem Mittelsmann Cordemann gehalten wurde. ${ }^{146}$ Cordemann, der damals das Berliner Büro der wirtschaftspolitischen Abteilung der ROL der NSDAP leitete, ${ }^{147}$ war gleichzeitig seit 1930 der Verbindungsmann Schleichers zur NSDAP und hatte dessen Verbindung zu Straßer hergestellt. $^{148}$

In diesen Begegnungen und Plänen des Frühjahres 1932 wird so überraschenderweise in Umrissen jene politische Konstellation erkennbar, auf die Schleicher ein halbes Jahr später, im Herbst 1932, seine Regierung zu gründen versuchte. Schon

140 Dalberg und Funk waren beide als Wirtschaftsjournalisten Mitarbeiter der „Berliner Börsenzeitung" gewesen.

141 Eberhard Czichon, Wer verhalf Hitler zur Macht? Köln 1967, S. 24.

142 Auskunft Dräger v. 31. 8. 1972.

143 Dräger, Entwurf.

144 Gereke, a. a. O., S. 162-174.

145 Grotkopp, a. a. O., S. 37.

146 Gereke, a. a. O., S. 173.

147 Tyrell, a. a. O., S. 362.

148 Brief Walter v. Etzdorf vom 26. 12. 1963, IfZ, ZS 1926 S. 4; Kurzorientierung des Ministeramtes vom 6. 9. 1932, IfZ, F 41; Auskunft Cordemann v. 8.9.1971. 
jetzt, als es noch um Pläne für die Arbeitsbeschaffung ging, trafen sich Vertreter des ADGB, bürgerliche Industrielle und Politiker sowie der Straßer-Flügel der NSDAP. Auch die Verbindung zu Schleicher bestand über Gereke und Cordemann bereits. Bei der engen Verflechtung und Zusammenarbeit der verschiedenen Gruppen ist es auch nicht verwunderlich, daß die Arbeitsbeschaffungspläne, die ausgearbeitet wurden, sich weitgehend ähnelten.

Die erste Arbeit war die von Friedländer-Prechtl, im Mai 1931 unter dem Titel „Wirtschaftswende“ veröffentlicht, die wiederum auf seiner Untersuchung von 1926 fußte. ${ }^{149}$ Im Januar 1932 präsentierten die Gewerkschaften ihren nach den drei Verfassern benannten WTB-Plan. ${ }^{150}$ Ebenfalls Anfang 1932 trat Wagemann mit seinem Plan an die Öffentlichkeit. ${ }^{151}$ Im März legte Dräger das Manuskript seiner Studie „Arbeitsbeschaffung durch produktive Kreditschöpfung“ vor. ${ }^{152}$ Gereke kam später mit ähnlichen Plänen heraus, die sich aber vor allem mit der verwaltungstechnischen Seite der Arbeitsbeschaffung befaßten. ${ }^{153}$ Dräger versuchte, die verschiedensten Regierungs- und Parteistellen, z. T. über die Wirtschaftsverbände, für seinen Plan zu gewinnen, ${ }^{154}$ doch gelang ihm das nur bei Straßer. Im Gegensatz zu den anderen Politikern und Fachleuten hatte sich Straßer seit Jahren mit Gedanken über eine auf Finanzreformen beruhenden aktiven Konjunkturpolitik zumindest nebenbei beschäftigt. Etwa seit Herbst 1931 war er dabei, auf der Grundlage seiner eigenen Ideen und der beginnenden Arbeit seiner Wirtschaftspolitischen Abteilung ein Wirtschaftsprogramm aufzustellen, das sich in ähnlichen Bahnen wie das der Reformergruppe bewegte. Bereits am 18. Dezember 1931 konnte die „Münchner Post" berichten, daß die Industrie auf Straßer setze, der als einziger ,ein realisierbares Wirtschaftsprogramm" habe. ${ }^{155} \mathrm{Da}$ er an dem Dräger-Plan über seine Mitarbeiter gleichsam selbst beteiligt gewesen und über jeden Entstehungsschritt informiert worden war, ist es nicht verwunderlich, daß er ihn im Prinzip sofort anerkannte und für die weitere Ausarbeitung seines eigenen Programms benützte. Im Frühjahr 1932 entstand auf dieser Grundlage ein Programm, dessen vorläufige Endfassung von Dr. von Renteln stammte, der damals in der ROL Leiter der Unterabteilung Währung, Finanzen und Produktion war. ${ }^{156}$ Straßer wurde so in die Lage versetzt, am 10. Mai 1932 in seiner Reichstagsrede die wichtigsten und werbewirksamsten Aspekte eines neuen NS-Wirtschaftsprogramms der Öffentlichkeit vorzustellen. Nach der für die Studiengesellschaft enttäuschenden Ablehnung, auf die das Dräger-

149 Siehe oben S. 103.

150 „WTB-Plan“, siehe Kroll, a. a. O., S. $403 \mathrm{ff}$.

151 „Wagemann Plan“; siehe Kroll, a. a. O., S. $396 \mathrm{ff}$.

152 Für diese Arbeit wurde die im Text unveränderte 4. Auflage, Düsseldorf 1955, benützt.

153 „Gereke-Plan“.

154 Dräger, a. a. O., S. $11 \mathrm{f}$.

155 Münchner Post, 18. Dez. 1931.

156 Brief Feder vom 15. 2. 1932 und Brief v. Rentelen vom 17.2. 1932 an die Reichsorganisationsleitung II, BA, NS 22/11. Dirk Stegmann, Zum Verhältnis von Großindustrie und Nationalsozialismus, in: Archiv für Sozialgeschichte, Bd. 13, (1973) S. 429. 
Programm, von Straßer abgesehen, sonst stieß, ließ Dräger seinen Plan unter dem Titel „Arbeitsbeschaffung durch produktive Kreditschöpfung“ noch 1932 zum Teil auf eigene Kosten drucken, und zwar im Juli als Heft 41 der NS-Bibliothek im Münchner Eher-Verlag und im August in politisch neutraler Form bei Hempel \& Co. in Berlin. Eine praktisch unveränderte Neuauflage erfolgte 1956 beim EconVerlag in Düsseldorf. Dräger selbst bezeugt, ${ }^{157}$ sein Programm sei unter Beratung von Dalberg ,in Anlehnung an Robert Friedländer-Prechtl, Dalberg, Woytinski (WTB-Plan) und das Gutachten des Reichswirtschaftsrates" entstanden. Dabei habe er versucht, auch alle Anregungen aus den Vorträgen bei der Studiengesellschaft (z. B. von Wagemann, Wildermuth, O. Chr. Fischer, Sombart) und Anregungen, die Dalberg und Grotkopp in einem Gespräch mit Keynes bekamen, in der Schrift zu verwerten. Er sah sich mit seinen konjunkturpolitischen Forderungen etwa in der Mitte zwischen dem vorsichtigeren WTB-Plan der Gewerkschaften und den kühnen, ,in einigen Teilen leicht ans Utopische“ grenzenden Plänen Friedländer-Prechtls; mehrfach bezog er sich auf Studien der Straßer-Mitarbeiter Daitz und Tholens. ${ }^{158}$

\section{Vergleich der Programme von Friedländer-Prechtl, Dräger und Straßer}

Kernstück der Arbeit von Dräger ist das Kapitel „Das Arbeitsbeschaffungsprogramm der gesteigerten Bedarfsdeckung", ${ }^{159}$ das in Inhalt und Aufbau sofort die Ähnlichkeit mit dem Straßer-Programm erkennen läßt. Auch bei Dräger liegt das Hauptgewicht auf Siedlungsmaßnahmen, wobei sowohl an die Städtesanierung gedacht ist, und zwar mit dem Aufbau landwirtschaftlicher Nebenerwerbsstellen im Einzugsgebiet der Industriestädte, wie auch an die Schaffung bäuerlicher Siedlungen vor allem im Osten. Dabei spielen für beide auch wehrpolitische Gedanken eine Rolle. Den Boden sollen die von Dräger wie von Straßer ausführlich dargelegten Landeskulturarbeiten beschaffen. Die sonstigen Maßnahmen, wie Städtebau, Elektrowirtschaft und Wasserstraßenbau, werden von beiden an den Schluß gestellt. Besonders auffallend ist die beiden gemeinsame Absage an eine Vergrößerung der Industrieanlagen, solche Fabrikationsbetriebe ausgenommen, die Ersatz für ausländische Rohstoffe herstellen. ${ }^{160}$ Deutlich ist auch die Übereinstimmung in den Zahlen: Z.B. fordern beide 100000 Siedlungen pro Jahr; Straßer glaubte, daß ein Eigenheim ohne Boden und Infrastruktur etwa 5000 RM koste, Dräger meinte, daß mit Boden und Infrastruktur $10000 \mathrm{RM}$ etwas zu niedrig seien, wies aber auf Tholens hin, der - wiederum ohne - auf $5000 \mathrm{RM}$ kam. ${ }^{161}$ Dräger schätzte die zu

157 Dräger, Entwurf ..., S. 9.

158 Ebenda. Daitz wird zitiert auf S. 88 und 91 zu Fragen des Wasserstraßenbaues und Berechnungen der Möglichkeit der Ausweitung des Kreditvolumens. Tholens wird zitiert auf S. 75, 79, 81 zu Fragen der Landeskulturarbeiten und der Siedlung.

159 Dräger, Entwurf ..., S. 69-97.

160 Ebenda, S. 89; Sofortprogramm, S. 14.

161 Dräger, Entwurf ... S. 79 Anm. 2. Tholens war Mitarbeiter Straßers. 
entwässernde Fläche auf 5-10 Millionen Hektar, Straßer auf 8,5 Millionen, wobei sie eine jährliche Ertragssteigerung von 400-780 Millionen RM bzw. 680 Millionen RM annahmen. Dräger rechnete mit Moor- und Ódlandflächen von 1-2 Millionen Hektar und einer Ertragssteigerung von 200-500 Millionen RM, Straßer mit 2,5 Millionen Hektar und 690 Millionen RM. Durch Flurbereinigung und ähnliche Maßnahmen glaubten beide etwa 5 Millionen Hektar verbessern zu können, was nach Dräger etwa 300 Millionen RM kosten und eine Ertragssteigerung von etwa 10-25\% erbringen würde, nach Straßer 500 Millionen bei Ertragsverbesserungen von $25-50 \%$. Die Gesamtkosten dieser Landeskulturarbeiten wurden von Dräger auf 7,5 Milliarden geschätzt, die jährlichen Investitionen auf etwa 1,215 Milliarden RM. Straßer kam auf Gesamtkosten von 9,5 Milliarden RM und auf Jahresraten von 1,5 Milliarden RM. ${ }^{162}$

Die geringen Abweichungen hinsichtlich dieser Zahlen kamen vor allem dadurch zustande, daß Dräger seine Arbeit für einen relativ kleinen Kreis geschrieben hatte, während Straßers Zahlen für die Öffentlichkeit bestimmt und deshalb häufig etwas zu schön gefärbt waren, z. B. wenn er die Kosten eines Eigenheims nur mit etwa 5000 RM annahm und dabei die Boden- und Infrastrukturkosten vergaß oder wenn er die Ertragssteigerungen durch Flurbereinigung mit 25-50\% weit überhöht angab. Ähnliches gilt auch für den sonst völlig gleichen Finanzierungsplan. Dräger wie Straßer wollten ihre Vorhaben zunächst aus Mitteln der Arbeitslosenversicherung und aus Steuer- und Versicherungsmehreinnahmen finanzieren und die Restsumme durch staatliche Kreditschöpfung bestreiten. Doch während Straßer, eingedenk der Inflationsfurcht bei den deutschen Wählern, die Kreditschöpfung mit $25 \%$ der Gesamtkosten etwas verharmloste, war Drägers Angabe von 50\% sicherlich realistischer. ${ }^{163}$

Über Grundlage und Herkunft der Ideen in Straßers Rede vom 10. Mai 1932, die für die damaligen wirtschaftspolitischen Debatten in Deutschland eine gewisse Zäsur setzte, sind schon mehrfach Überlegungen angestellt worden. So wies Kroll, der die Bedeutung der Rede und des Straßer-Programms als einer der ersten erkannt hat, auf die überraschenden Ähnlichkeiten mit den Ideen Friedländer-Prechtls hin, ${ }^{164}$ wobei er glaubte, daß Friedländers Überlegungen Straßer eher zufällig in die Hände gefallen seien, er ihren Wert erkannt und sie, indem er praktisch geistigen Diebstahl beging, als die seinen politisch verwertet habe. ${ }^{165}$ Nun trifft es zwar zu, daß Friedländer-Prechtl mit seinen Veröffentlichungen den Anstoß und damit auch wesentliche Grundlagen für die Diskussion und die Arbeiten der Studiengesellschaft geliefert hat. Trotzdem läßt sich beim Vergleich aller drei Programme leicht nachweisen, daß das Straßer-Programm mehr mit der Drägerschen Arbeit übereinstimmt

162 Sofern die Zahlenangaben in der Straßerrede vom 10.5. 1932 fehlen, sind sie hier durch solche aus dem Sofortprogramm vom Juli 1932 ergänzt worden.

163 Kroll, a. a. O., S. 432.

164 Ebenda, S. $435 \mathrm{ff}$.

165 Ebenda, S. 455. 
als mit dem Entwurf Friedländer-Prechtls, der im 14. Kapitel seines Buches „Die Wirtschaftswende" 166 wohl ebenfalls Vorschläge zur Arbeitsbeschaffung machte, bei denen er die Meliorationen an erster Stelle nannte, jedoch im Gegensatz zu Dräger und Straßer keine Zahlenangaben oder Größenordnungen bot. Friedländer-Prechtl wurde bei der Stickstoffindustrie und beim Autobahnnetz konkreter, bei Punkten, die wiederum Dräger und Straßer nur kurz streiften.

Die Benzingewinnung aus Kohleverflüssigung, in der „Wirtschaftswende“ unter Angabe konkreter Zahlen gefordert, ist bei Dräger und Straßer nur noch in allgemeiner Form zu finden, nämlich in der Forderung nach planmäßigem Ausbau der Industrien, die von ausländischen Rohstoffen unabhängig machen. Andererseits fehlt bei Friedländer-Prechtl eine genau ausgeführte und mit Zahlen versehene Lieblingsidee Drägers und Straßers, nämlich die Eigenheimsiedlung zur Stadtsanierung und die Ostsiedlung. Der ursprüngliche Plan Friedländer-Prechtls war also durch die intensive Arbeit der Studiengesellschaft beträchtlich verändert und weiterentwickelt worden. Dabei ergab sich einerseits eine beginnende Präzisierung, auf der anderen Seite stellten sich Unterschiede ein, die durch verschiedene Wissens- und Interessenlagen bedingt waren.

Ursula Hüllbüsch hat zur Entstehung des Straßerprogramms von 1932 die Ansicht vertreten, ${ }^{167} \mathrm{da} \beta$ es von Mitgliedern des ,"Tat"-Kreises, vor allem von Elbrechter, ${ }^{168}$ verfaßt worden sei. Sie stützt sich auf eine Mitteilung Elbrechters vom 9. März 1955 und auf Formulierungsvergleiche. Am bedeutsamsten scheint ihr eine Stelle, in der Straßer begründete, weshalb er eine Unterscheidung zwischen SPD und ADGB für notwendig halte: Die Führung der SPD sei ,teilweise fremdrassig intellektuell beeinflußt “, ,während die Führer der deutschen Gewerkschaft ... von alters her und auch heute noch aus dem Arbeiterstand hervorgegangen sind“. ${ }^{169}$ Diese Stelle setzt Hüllbusch mit einem bereits im März 1932 in der „Tat" erschienenen Artikel in Parallele, in dem es heißt: ,,... die Gewerkschaften, die als ständische Gruppierung auf einem viel festeren Fundament stehen als die Partei und in ihrer Führung nicht in dem Maße von liberalistischen Intellektuellen korrumpiert wurden, ..."170 Diese These widerspricht der hier beschriebenen Entstehungsgeschichte des Straßer-Programms nicht grundsätzlich. Schließlich war Ferdinand Fried, der Wirtschaftsfachmann des „Tat"-Kreises, ebenfalls, wenn auch nur am Rande, an den Arbeiten der „Studiengesellschaft“ beteiligt. Er dürfte also Kenntnis von den Überlegungen der

166 Friedländer-Prechtl, Die Wirtschaftswende, S. $146 \mathrm{ff}$.

167 Ursula Hüllbüsch, Gewerkschaften und Staat, Phil. Diss., Heidelberg 1961. Vgl. auch Hannes Heer, Burgfrieden oder Klassenkampf, Neuwied/Berlin 1971, der die Argumentation von Hüllbüsch übernommen hat.

168 Elbrechter, geb. 19. 1. 1895, Studium der Zahnheilkunde, Mitarbeiter von Straßer und Kaufmann 1925/26 in Elberfeld, beteiligt am Bamberger Programm, seit 1923 freier Mitarbeiter der „Tat", befreundet mit Straßer, Zehrer, Schleicher, Brüning und Treviranus.

168 Verhandlungen des Reichstages, Bd. 446, S. 2512b + c.

170 Die Tat, 1931/32, Bd. II, S. $1025 \mathrm{f}$. 
Gesellschaft und sicher auch von dem Drägerschen Entwurf gehabt haben. Im übrigen war Elbrechter, auf den Hüllbüsch hier besonders verweist, ein alter Bekannter, seit Herbst 1931 sogar zunehmend enger Vertrauter und politischer Berater Straßers. Es ist wahrscheinlich, daß er Straßer bei der Formulierung der Reichstagsrede vom 10. Mai 1932 behilflich war; die ROL dürfte Straßer einen Plan vorgelegt haben, der erst noch in eine Parlamentsrede übertragen werden mußte. Daß der Straßer-Plan dem ,Tat"-Kreis bereits vorher bekannt war, ist auch daraus ersichtlich, daß in der gleichen Märzausgabe der „Tat" ein mit F. F. gezeichneter Artikel erschien, der den WTB-Plan des ADGB kommentierte und dabei erwähnte, dieser Plan stehe dem Wirtschaftsdenken der NSDAP sehr nahe, ${ }^{171}$ obwohl die NSDAP ihre Gedanken ja erst mit der Straßer-Rede vom 10. Mai 1932 der Öffentlichkeit vorstellte.

Auch Walther Funk erhob später Anspruch darauf, an der Ausarbeitung des Programms maßgeblich beteiligt gewesen zu sein. ${ }^{172}$ In der Tat hatte Funk ebenfalls losen Kontakt zur „Studiengesellschaft“. Außerdem war er wirtschaftspolitischer Berater der ROL und mit Straßer persönlich befreundet. Er hat daher sicherlich sowohl an wirtschaftspolitischen Vorstudien der ROL als auch an der Endfassung der Reichstagsrede mitgewirkt.

\section{Weitere wirtschafts- und sozialpolitische Forderungen Straßers}

Neben dem Skelett einer auf den Ideen der Reformgruppen basierenden antizyklischen Konjunkturpolitik, das in Straßers Wirtschafts- und Arbeitsbeschaffungsprogramm erkennbar ist, fallen noch einige Vorschläge auf, die ebenso wie die wirtschaftspolitischen Grundideen eine auf dem „Bamberger Programm“ fußende „Straßersche Richtung“ im Nationalsozialismus erkennen lassen. ${ }^{173}$ Die staatliche Lenkung der Wirtschaft wurde von Straßer bejaht. ${ }^{174}$ So forderte er, daß ,Monopole, die nicht aufgelöst werden können“ - was er allerdings nicht genauer definierte -, ,,sofort in unmittelbare staatliche Verwaltung genommen werden“, d. h. daß sie „verstaatlicht" werden. Er begründete dies damit, daß ein Monopol „vom Staat ebenso gut verwaltet werden" könne „wie von der Privatwirtschaft. Die staatliche Verwaltung garantiert aber, daß die Gewinne der Allgemeinheit zugute kommen und nicht dem Finanzkapital". Die übrigen Aktiengesellschaften sollten wie die Banken unter „Staatsaufsicht" gestellt werden. Die „Staatsaufsicht" wiederum, die bei den Banken nur als erster Schritt zu einer späteren Verstaatlichung anzusehen sei, gliederte er in das Aufsichts- und Eingriffsrecht des Staates einerseits und die

171 Ebenda, S. 1027.

172 Prozeß gegen die Hauptkriegsverbrecher, Bd. XIII, S. 97, Auskunft Horst Walter v. 18. 11. 1971.

173 Siehe oben S. 99f.

174 Sofortprogramm, S. 11 und $14 \mathrm{f}$. 
Berichterstattungspflicht der Banken bzw. der Aktiengesellschaften andererseits: „Das Aufsichtsrecht muß dem Staat die Möglichkeit geben, sich über jedes Vorkommnis auf dem Gebiete des Bankwesens (bzw. der Aktiengesellschaften) von den Beteiligten volle Klarheit verschaffen zu können, das Eingriffsrecht ermöglicht, die im Interesse der Volkswirtschaft erforderlichen Maßnahmen auf dem Gebiet des Bankwesens (der Aktiengesellschaften) durchzuführen. Die Berichterstattungspflicht hat in einer monatlichen Offenlegung aller Positionen und wichtigen Veränderungen gegenüber dem Aufsichtsamt zu bestehen.“

Er glaubte, daß ,erst durch diese Maßnahmen ... die Möglichkeit zur Verhinderung von Fehlinvestitionen geschaffen“" werde und durch „Brechung der Zinsknechtschaft" die „Voraussetzungen zur Finanzierung des Arbeitsbeschaffungsprogramms" entstünden.

Straßers Urteil über die deutsche Volkswirtschaft wurde nicht zuletzt von der Ansicht bestimmt, daß sie ,,an einer Aufblähung des industriellen Produktionsapparates und an einer zu geringen Entwicklung unserer Landwirtschaft" leide. Neben der im gesamten völkischen Lager vorhandenen Vorliebe für eine agrarisch-ländliche Wirtschaftsstruktur wird hier auch einmal kurz sichtbar, wie Straßer über die Ursachen der Krise dachte. Offenbar glaubte er, daß die Wirtschaftsentwicklung der letzten Jahrzehnte eine ungesunde Verschiebung von der Landwirtschaft zur Industrie gebracht habe und die dadurch entstandene „Disproportionalität“ als Krisenursache anzusehen sei, womit er wieder auf die alten liberalistischen Bilder zur Konjunkturerklärung zurückgriff. ${ }^{175}$ Jedenfalls forderte er, daß ,,neugebildete Kapitalien nicht in der industriellen, sondern in der landwirtschaftlichen Produktion angelegt werden. Die Neuerstellung industrieller Produktionsanlagen muß genehmigungspflichtig gemacht werden. Die Genehmigung wird nur beim Vorliegen besonderer Gründe erteilt werden (z. B. für Erstellung von Werken, die Rohstoffzufuhr entbehrlich machen)".

In einem Abschnitt über ,,soziale Fragen“ ${ }^{176}$ hat Straßer nicht allein das Recht auf Arbeit proklamiert, sondern auch die Sicherung der Sozialversicherung und ,den großzügigen Ausbau der Altersversorgung" verlangt. Ebenso faßte er die Gewinnbeteiligung ins Auge: „Zinssenkung ..., Preiskontrolle ..., Staatsaufsicht über die Aktiengesellschaften ..., das Gesetz der Arbeit ..., die steuerlichen Maßnahmen ... (vor allem stärkere Besteuerung der höheren Einkommen, d. Verf.) und die Sparmaßnahmen ... bürgen dafür, daß alle Erträge in erster Linie der Belebung der deutschen Wirtschaft durch die Arbeitsbeschaffung dienstbar gemacht werden. Sobald die deutsche Wirtschaft durch die Arbeitsbeschaffung wieder in den Stand gesetzt ist, nennenswerte Gewinne abzuwerfen, wird die Zeit zu einer umfassenden Regelung der Gewinnbeteiligung gekommen sein."

Zum Schluß ging Straßer noch auf den Arbeitsdienst ein. Mit Recht betonte er, daß der Arbeitsdienst aus konjunkturellen Gründen nicht in Konkurrenz zur freien

175 Vgl. Kroll, a. a. O., S. 434.

176 Sofortprogramm, S. 16. 
Wirtschaft treten dürfe, vielmehr solle er zum Ziele haben: „Arbeiten auszuführen, die für die Belebung der gesamten Wirtschaft notwendig sind und auf dem normalen Wege nicht durchgeführt werden können. (Die Arbeitsdienstpflichtigen werden also den Arbeitern, die in Tariflohn stehen, keinerlei Konkurrenz machen.)“"177 Wichtig sei, daß alle jungen Männer ohne Rücksicht auf Stand oder Besitz eingezogen würden und auf diese Weise die Achtung vor der Handarbeit lernten. Der Arbeitsdienst war im Sinne Straßers ,das große Erziehungsmittel der Zwanzigjährigen, auf dem Asphalt der Straße heimatlos gewordenen deutschen jungen Menschen. Körperliche Arbeit erzieht den Respekt vor der Handarbeit, überwindet Klassendünkel und Klassenhaß und stellt über die Heimatscholle hinweg die Verbindung mit dem deutschen Staatsgedanken wieder her." 178

Abgesehen von der im Sinne der früher behandelten Konjunkturtheorie ${ }^{179}$ richtigen Bemerkung, daß die Wirtschaft Vertrauen zum Staat und seiner Entwicklung haben müsse, um florieren zu können, ${ }^{180}$ sagt Straßer nichts über die Ursachen der Weltwirtschaftskrise; insofern ist das Straßersche Programm enttäuschend. Wohl aber ist seine Krisentherapie, d. h. ,,die Arbeitsbeschaffung zum normalen Lohn“, finanziert durch ,produktive Kreditschöpfung“, ${ }^{181}$ als im wesentlichen richtig anzuerkennen; sie erwies sich, wie noch zu beschreiben sein wird, auch als durchführbar. Mit seinem Wirtschaftsprogramm setzte sich Straßer aber, wiewohl es ob seines konstruktivsachlichen Inhaltes Aufsehen erregte und Anerkennung fand, nicht nur zu anerkannten ökonomischen Lehrmeinung in Widerspruch, sondern auch zur Wirtschaftspolitik der Regierung Brüning.

\section{Exkurs: Die Wirtschaftspolitik Brünings}

Brüning hat zwar mit einer Vielzahl von Maßnahmen, im Laufe der Krise mit steigender Häufigkeit durch Notverordnung, aktiv in die Wirtschaftspolitik eingegriffen und sich somit formal in Gegensatz zur verbreiteten klassischen Lehre von den Selbstheilungskräften der Wirtschaft gesetzt. Jedoch widersprachen die Notverordnungen ${ }^{182}$ prinzipiell nicht der liberalistischen Wirtschaftsauffassung, griff die Regierung doch nur dort ein, wo der Anpassungsprozeß von Kosten und Preisen, Angebot und Nachfrage aufgrund - gemäß der liberalen Lehre ungerechtfertigter - Einschränkungen der Beweglichkeit nicht ohne weiteres möglich war, z. B. bei den

177 Ebenda.

178 Gregor Straßer, Rundfunkrede vom 29.7. 1932, Deutsches Rundfunkarchiv, Frankfurt, Tonband VII 20004/86.

179 Siehe oben S. 89 ff.

180 Verhandlungen des Reichstages, Bd. 446, S. 2513b.

181 Interne Ausarbeitung Straßers für Reichs- und Landtagsanträge vom 24. 8. 1932, BA, NS $22 / 348$.

182 Hierbei ist eine Ausnahme bei den Notverordnungen anläßlich der Bankenkrise zu machen. 
durch Tarife festgelegten Löhnen und Gehältern, bei Mieten, die meist vertraglich gebunden sind, bei den Preisen der Kartellgüter oder den gebundenen Preisen der Markenartikel. Über die Bewertung dieser oft unter dem Stichwort „Deflationspolitik" zusammengefaßten Wirtschaftspolitik Brünings herrscht ein Streit, der weitgehend aus den völlig unterschiedlichen Ansätzen zu erklären ist. Die modernen Ökonomen, wie etwa Kroll, urteilen vom heutigen Wissensstand aus durchaus richtig, wenn sie die damals relevanten Faktoren des Wirtschaftsablaufes ins Verhältnis zu den Maßnahmen der Politik setzen und dabei klar erkennen, daß die Maßnahmen falsch waren und häufig sogar das Gegenteil dessen bewirkten, was sie beabsichtigten. ${ }^{183}$ Indem sie den historischen Kontext außer acht lassen, zu dem neben den außenpolitischen Gegebenheiten, etwa der Frage der Reparationen oder der Abhängigkeit vom Welthandel, auch innenpolitische Machtverhältnisse und die damals vorhandene Erkenntnis wirtschaftlicher Zusammenhänge gehörten, kommen sie zu im Sinne der historischen Forschung schiefen Ergebnissen. Anders verhält es sich bei den Nicht-Ökonomen, die sich bei ihrem Urteil auf die zeitgenössische wirtschaftswissenschaftliche Literatur stützen und deshalb die Problematik der Brüningschen Wirtschaftspolitik kaum zu sehen vermögen ${ }^{184}$ oder annehmen, Brüning habe seine „falsche" Wirtschaftspolitik durchaus durchschaut, sie aber zur Erreichung des Endes der Reparationen als außenpolitisches Mittel eingesetzt, um dann auf Wirtschaftsankurbelung und Arbeitsbeschaffung umzuschalten, wie etwa Helbich meint. ${ }^{185} \mathrm{Um}$ zu einer einigermaßen richtigen Beurteilung der damaligen Maßnahmen zu kommen, muß aber sowohl der Wissensstand der modernen Konjunkturforschung herangezogen als auch die damalige politische Situation berücksichtigt werden, wie dies in fundierter Weise durch Sanmann geschehen ist. ${ }^{186}$ Hier wird die Wirtschafts- und Finanzpolitik Brünings in drei sachbezogene Teilbereiche gegliedert: die Deflationspolitik, die Parallelpolitik und die Agrarpolitik. Unter Deflationspolitik sind hier die Versuche der Regierung zu verstehen, zunächst durch Appelle, dann durch Notverordnungen das innerdeutsche Preisniveau auf das Weltmarktniveau zu senken. Das Stichwort „Parallelpolitik“ umreißt den Versuch, auch bei kunjunkturbedingt sinkenden Staatseinnahmen den Haushalt auf jeden Fall auszugleichen, indem einerseits neue Steuereinnahmen erschlossen werden und andererseits Ausgaben, z. B. Gehälter, Pensionen und die Erwerbslosenunterstützung, im Haushalt gekürzt werden. Beide Teilmaßnahmen hatten jede für sich depressionsverschärfende Wirkungen, die sich gegenseitig noch verstärkten. Die Deflationsmaßnahmen bewirkten, da es praktisch kaum möglich war, alle Kosten und Preise gleichzeitig zu senken, Verzerrungen der Erlös-Kosten-Relation für viele Branchen,

\footnotetext{
183 Kroll, a. a. O., S. $361 \mathrm{ff}$.

184 Bracher, a. a. O., S. $435 \mathrm{ff}$.

185 W. J. Helbich, Die Reparationen in der Ära Brüning. Zur Bedeutung des Young-Planes für die deutsche Politik 1930-32, Berlin 1962, S. 54, 59.

186 Horst Sanmann, Daten und Alternativen der deutschen Wirtschafts- und Finanzpolitik in der Ära Brüning, in: Hamburger Jahrbuch für Wirtschafts- und Gesellschaftspolitik 10 (1965), S. 109-140.
} 
die zu Produktionseinschränkungen führten. Zum anderen wurde durch die Erwartung weiteren Preisverfalls die Investitionsneigung der Unternehmen, aber auch der Privathaushalte, noch mehr geschwächt. Die Parallelpolitik im Wirtschaftsabschwung erhöhte durch neue Steuern einerseits die Kosten in vielen Bereichen, verlangsamte aber vor allem durch Senkung der Haushaltsgröße die Kreislaufgeschwindigkeit des Geldes und wirkte so ebenfalls depressionsverschärfend. Die Agrarschutzpolitik war weitgehend konjunkturneutral und hatte sogar auf landwirtschaftlichem Sektor einige belebende Effekte, die jedoch zu schwach waren, den Niedergang der Landwirtschaft in der allgemeinen Wirtschaftskrise aufzuhalten. Werden nun diese Maßnahmen in Zusammenhang mit den Zielen der Politik Brünings gebracht, die ab Ende 1930 im wesentlichen auf die Ablösung der Reparationen gerichtet war, so ergeben sich folgende Zusammenhänge. Die Reparationen brachten zwei Probleme mit sich, zunächst das Aufbringungsproblem, dann - soweit es sich nicht um Sachleistungen, sondern um Devisen- bzw. Goldzahlungen handelt - das Transferproblem. Das Aufbringungsproblem, das durch steigende Raten bis 1988 noch erschwert wurde, verlangte eine langfristig stark wachsende Wirtschaft. Für das Transferproblem, das für den Devisenanteil der Zahlungen entstand, gab es mehrere Lösungen, falls die Beschaffung der Devisen aus Exportüberschüssen nicht unmittelbar möglich war. Einmal bestand die Möglichkeit von langfristigen Auslandsanleihen, wie bereits in den zwanziger Jahren vorexerziert. Dann hätten die Zahlungen in Warenlieferungen überführt oder die aufgebrachten Leistungen thesauriert werden können, bis sich später eine Möglichkeit der Transferierung ergeben hätte. Nur langfristig, d. h. bis 1988 oder sogar noch darüber hinaus, hätte die Transfersumme durch Exportüberschüsse ausgeglichen werden müssen. Durch seine auf Exportförderung zielende Deflationspolitik versuchte nun Brüning fast verzweifelt, wenn auch erfolglos, die Devisen zu erwirtschaften, mit denen er das Transferproblem lösen wollte, aber durch eben diese Deflationspolitik, gekoppelt mit der Parallelpolitik, trug er andererseits zum starken Rückgang der Wirtschaft bei, wodurch er das an und für sich primäre Problem der Aufbringung nahezu unlösbar machte. Sein erklärtes Ziel war, sich als gutwilliger Reparationsschuldner zu erweisen, der die größten Anstrengungen machte, seinen Zahlungsverpflichtungen nachzukommen. Gerade dabei, so glaubte er, werde sich erweisen, daß die Reparationsleistungen für Deutschland untragbar seien, was dann zu ihrem Ende führen werde. Dieses außenpolitische Ziel erreichte er auch, allerdings dadurch, daß er mit einer Politik, die deutsche Zahlungswilligkeit beweisen sollte, die deutsche Wirtschaftskraft noch weiter schwächte und die völlige Zahlungsunfähigkeit noch schneller herbeiführte. Es wäre aber falsch zu glauben, daß gerade darin die Raffinesse und Klugheit Brüningscher Politik bestanden habe; eine Voraussetzung wäre ja, daß nur Brüning und seine Ratgeber diese wirtschafts- und finanzpolitischen Zusammenhänge erkannt, alle anderen, insbesondere die Fachleute der ausländischen Gläubigerstaaten, sie jedoch nicht bemerkt hätten. Indes wurde Brüning bis zum Ende seiner Regierung sowohl vom Ausland als auch vom Inland bescheinigt, daß allein seine Politik den Bestimmungen des Youngplanes entspreche; auch offizielle inter- 
nationale Sachverständige haben das, z. B. im „Layton-Bericht“ und im „BeneduceBericht" ausdrücklich bestätigt. ${ }^{187}$

Die Ansicht, daß Brüning nach der Lösung der Reparationsfrage, die er ja nicht mehr im Amte erlebte, auf eine aktive Konjunkturpolitik zur Arbeitsbeschaffung umschalten wollte, ${ }^{188}$ kann in dieser Form ebenfalls nicht aufrecht erhalten werden. Die geplanten Arbeitsbeschaffungsmaßnahmen, die von der Ministerialbürokratie vorbereitet wurden, ${ }^{189}$ sollten nur aus Mitteln eines ausgeglichenen Staatshaushalts finanziert werden, was keine substantielle Nachfrageerhöhung bewirkt und damit die Konjunktur nicht stimuliert hätte. ${ }^{190}$ Die vorbereiteten Pläne bezogen sich insbesondere auf den freiwilligen Arbeitsdienst ${ }^{191}$ und sind vor allem als psychologisches Trostpflaster und Ablenkungsmanöver für die Bevölkerung gedacht gewesen, um unpopuläre Maßnahmen wie etwa eine Kürzung der Sozialausgaben besser durchsetzen zu können. ${ }^{192}$ Ein antizyklisches Wirtschaftsprogramm ist von der Regierung Brüning im Zusammenhang mit dem absehbaren Ende der Reparationen nicht einmal in Ansätzen entwickelt worden. Es blieb auch ohne jeden Einfluß auf die Brüningsche Regierungspolitik, daß auf einer Ressortbesprechung im Reichsfinanzministerium am 12. Februar 1932 Reichsfinanzminister Dietrich seinen Oberregierungsrat Lautenbach ${ }^{193}$ über Finanzierungsmöglichkeiten eines Arbeitsbeschaffungsprogramms referieren ließ und daß Lautenbach, einer der eifrigsten Reformer, deutlich eine Kreditausweitung und damit eine grundsätzliche Neuorientierung der Brüningschen Wirtschaftspolitik forderte: „Die Mittel zur Finanzierung öffentlicher Aufträge dürfen nicht anderweitigen produktiven Verwendungsmöglichkeiten, z. B. durch Besteuerung der Wirtschaft entzogen werden. Eine Ankurbelung der Wirtschaft ist nur möglich, wenn es sich nicht um eine Kaufkraftverlagerung, sondern um die Schaffung zusätzlicher Kaufkraft durch Kreditausweitung handelt."194

Brüning erreichte zwar das ihm vor Augen liegende Ziel, das Ende der Reparationen. ${ }^{195}$ Aber die aus seiner Sicht einheitliche Wirtschafts-, Finanz- und Außenpolitik war aufgrund mangelnder Einsicht in die ökonomischen Bedingtheiten der Krise letztlich falsch. Sie führte nur deshalb zum außenpolitischen Erfolg, weil die Gläubigerstaaten den gleichen wirtschaftspolitischen Trugschlüssen unterlagen und Brü-

187 Sanmann, a. a. O., S. 134.

188 Helbich, a. a. O., S. 59 f.; Hans Luther, Vor dem Abgrund. Reichsbankpräsident in Krisenzeiten, Frankfurt/Berlin 1964, S. 256.

188 Helbich, a. a. O., S. 60 f.; Auskunft Schwerin-Krosigk v. 28. 11. 1971.

190 Köhler, a. a. O., S. $304 \mathrm{f}$.

191 Köhler, a. a. O., S. $276 \mathrm{ff}$;; vgl. auch derselbe, Sozialpolitik von Brüning bis Schleicher, in: VfZ 21 (1973), S. $146 \mathrm{ff}$.

192 Köhler, a. a. O., S. 280f., Dietmar Petzina, Elemente der Wirtschaftspolitik in der Spätphase der Weimarer Republik, in: VfZ 21 (1973) S. $127 \mathrm{ff}$.

193 Vgl. Kroll, a. a. O., S. 380 ff.; Dräger, a. a. O., S. 21 f.; Grotkopp, a. a. O., S. 22 f.; Ohl, a. a. O., S. 64.

195 Köhler, a. a. O., S. 279 f.

195 Das Ende der Reparationen wurde erst unter der Regierung von Papen auf der Konferenz von Lausanne am 10. Juli 1932 erreicht. 
nings Politik als in ihrem Sinne richtig ansahen. Diese Politik des Primats der AuBenpolitik bewirkte jedoch durch die Fehleinschätzung der konjunkturellen Zusammenhänge eine derartige Verschlimmerung der wirtschaftspolitischen Situation in Deutschland, daß sich die Weimarer Republik, in der noch in obrigkeitsstaatlichen Kategorien denkenden Bevölkerung nur unvollständig verankert, im Chaos der Wirtschaftsdepression auflöste und der braunen Diktatur anheimfiel.

Tragisch ist auch, daß die Ideen der Reformer, die den Beginn einer neuen Konjunkturtheorie anzeigten, weder von Brüning noch von einem der anderen Politiker der Weimarer Parteien in ihrem Wert erkannt und aufgegriffen wurden. Allein Gregor Straßer, den die Entwicklung seines eigenen Denkens dafür prädestinierte, unterstützte die neuen Ideen, und im Frühjahr 1932 faßte er sie in ein politisch schlagkräftiges Programm und gab damit der NSDAP einen agitatorischen Vorsprung.

\section{Die Propagandawirkung des Straßer-Programms}

In einer Situation, in der über 6 Millionen Arbeitslose ${ }^{196}$ und ein Heer von Kurzarbeitern die innenpolitische Szene beherrschten, und auch diejenigen Arbeiter, Angestellten und mittelständischen Selbständigen, die noch Arbeit hatten und sich in einer einigermaßen erträglichen Einkommenslage befanden, sich ständig fragen mußten, wann auch sie ohne Arbeit und Auskommen sein würden, war nichts so zugkräftig, wie ein Arbeitsbeschaffungsprogramm. Kein außenpolitischer Erfolg konnte, wie Brüning glaubte, die bürgerlichen Schichten gewinnen, auf die er sich vor allem stützen wollte, solange es nicht gelang, breiten Schichten bessere ökonomische Aussichten zu bieten. Solche Erwartungen konnten sich aber nur an konkreten oder glaubwürdig angekündigten und in jedem Falle einsichtigen Maßnahmen entzünden. Und eben solche Maßnahmen schlug Straßer vor. Landeskulturarbeiten, Eigenheimsiedlungen oder Straßenbau waren Projekte, von deren Notwendigkeit breite Volksschichten überzeugt werden konnten. Daß diese Arbeiten dem Gemeinwohl nützen würden, daß in Deutschland für sie genügend Arbeitskräfte zur Verfügung standen, daß auch die industriellen Voraussetzungen sowohl zur Durchführung der Arbeiten selbst wie zur Befriedigung der Bedürfnisse der durch jene Projekte wieder in Arbeit und Lohn kommenden Arbeitskräfte vorhanden waren, leuchtete unmittelbar ein. Straßers Slogan: „Nicht Kapital schafft Arbeit, sondern Arbeit schafft Kapital!“197 zündete.

Die Reichstagsrede vom 10. Mai 1932 wurde in der NS-Presse und in der gegnerischen Presse so lebhaft kommentiert, daß Straßers Programm sofort weiteste Verbreitung fand. Kurz danach wurde es unter dem Titel „Wirtschaftliches Sofortpro-

196 Im Jahresdurchschnitt 1932 gab es 5,6 Millionen bei den Arbeitsämtern gemeldete Arbeitslose; vgl. Kroll, a. a. O., S. 109.

197 Verhandlungen des Reichstages, Bd. 446, S. 2514 a. 
gramm" in stichpunktartiger Form als Rednermaterial (Auflage $600000^{198}$ ) zur Vorbereitung der Reichstagswahlen vom 31. Juli 1932 veröffentlicht. Es war in ähnlicher Weise zusammengestellt wie das seit dem 1. Juli 1929 von Fritz Reinhardt, dem Reichspropagandaleiter II, vierzehntägig herausgegebene Rednermaterial. ${ }^{199}$ Doch diesmal zeichnete nicht Reinhardt verantwortlich, sondern Gregor Straßer selbst. Es hieß im Vorspann:

\section{„Wirtschaftliches Sofortprogramm der NSDAP“}

Ausgearbeitet von der Hauptabteilung IV (Wirtschaft) der Reichsorganisationsleitung der NSDAP

Die nachfolgende Aufstellung enthält das wirtschaftliche Sofortprogramm der NSDAP in der Form eines knappen Rednermaterials. Es gilt als verbindliche Richtschnur für die Redner der NSDAP, sowie für die Veröffentlichungen in der Presse. Alle Äußerungen von Parteigenossen, die von diesem Material abweichen oder ihm widersprechen, sind lediglich als private Meinungsäußerung zu betrachten.

Den Rednern der NSDAP wird das Material insbesondere für den Wahlkampf zur Reichstagswahl vom 31. Juli 1932 empfohlen.

\section{Gregor Straßer \\ Reichsorganisationsleiter der NSDAP}

$\mathrm{Da} ß$ ein solches Programm überhaupt entstehen und auch noch für die ganze Partei verbindlich werden konnte, ist erstaunlich. Zum ersten Mal seit jenem an Hitlers Widerstand gescheiterten Bamberger Programm von 1926 war innerhalb der NSDAP wieder eine programmatische Aussage gemacht worden, und zwar diesmal eine Aussage, die offensichtlich von Hitler stillschweigend anerkannt wurde. ${ }^{200} \mathrm{Be}$ zeichnend für die Machtverhältnisse in der Partei war ferner, daß dieses Programm weder von Hitler selbst noch vom offiziellen Parteiprogrammatiker Feder stammte, und auch nicht, obwohl es als Propagandamaterial gedacht war, von Goebbels, dem Reichspropagandaleiter I, herausgegeben wurde, sondern von Gregor Straßer und seiner Reichsorganisationsabteilung erarbeitet worden war. Neben den unzähligen Rednern in der Partei, die in diesem Wahlkampf auftraten und sich vor allem auf dieses Sofortprogramm beriefen, verbreitete es Straßer selbst in zahlreichen großen

197 Finanziert wurde diese Auflage von Dr. Dräger, Auskunft Dräger v. 31. 8. 1972.

199 Siehe oben S. 58.

200 Dirk Stegmann, a. a. O., S. 429 ff. Stegmann arbeitet hier deutlich den Widerstand Hitlers gegen dieses Straßerprogramm heraus. Hierin wird Hitler sowohl von Schacht als auch vom „Keppler-Kreis“ und der damit verbundenen Industrie unterstützt (Vgl. Henry Ashby Turner jr, Faschismus und Kapitalismus in Deutschland, Göttingen 1972, S. 18). Überbewertet wird dieser Widerstand Hitlers allerdings, wenn angenommen wird, daß das Programm daraufhin zurückgezogen worden sei, wie es etwa Schacht in einem Brief an Industrielle vom 12. September 1932 meldete (Stegmann, a. a. O., S. 465; Turner, a. a. O., S. 18) Im Gegenteil ist aus den Akten der ROL klar zu ersehen, daß dieses Programm bis Dezember 1932 von Straßer weiterverfolgt wurde und auch als parteiverbindliche Erklärungen in die NS-Zeitungen und in die Parlamente als Anfrage eingebracht wurde, ohne daß hierbei der Widerstand Hitlers in irgendeiner Form deutlich geworden wäre. 
Reden. In seinen beiden Rundfunkreden vom 14. Juni 1932 und 29. Juli 1932, die auch als Schallplatten erschienen, ${ }^{201}$ konnte er bereits davon ausgehen, daß sein Arbeitsbeschaffungsprogramm in der Öffentlichkeit bekannt war und er daher schon mit den Begriffen seiner Wirtschaftspolitik arbeiten konnte, ohne sie näher erläutern zu müssen. ${ }^{202}$ Diesen beiden Rundfunkreden kommt auch deshalb Bedeutung zu, weil es sich um die ersten Sendezeiten handelte, die der NSDAP zur Verfügung gestellt wurden. Aber nicht etwa Hitler oder gar Goebbels oder Göring traten dabei als Redner auf, sondern beide Male präsentierte sich Gregor Straßer als Vertreter der NSDAP ${ }^{203}$ - ein Indiz sowohl für seine damalige Machtstellung in der Partei als auch für die Werbewirksamkeit seiner Ideen. In den Wählermassen entstand der Eindruck, daß neben der fast mythischen Führerfigur der Bewegung, Adolf Hitler, Gregor Straßer der Mann der praktischen Politik sei. Der Wahlerfolg der NSDAP vom 31. Juli 1932, der sie mit 230 Mandaten zur stärksten Reichstagsfraktion machte, ist in der Tat ohne Straßers Wirtschaftsprogramm nicht in dieser Höhe denkbar. Allein er - und damit die NSDAP - boten den verunsicherten Schichten des Mittelstandes und auch den Arbeitslosen ein allgemein einsichtiges und verständliches Programm, und die NSDAP nutzte diese propagandistische Möglichkeit voll aus. ${ }^{204}$ Indes wurde in der wirtschaftspolitischen Abteilung der ROL an den Plänen zur Krisenbekämpfung ständig weitergearbeitet. Im August 1932 kamen diese Arbeiten zu einem gewissen Abschluß, was sich darin zeigte, daß Straßer als Reichsorganisationsleiter am 24. August den entsprechenden Parteigliederungen und Fraktionen ausgearbeitete Pläne und Anträge für den Reichstag und für die Landtage zuleitete. ${ }^{205}$ In den Anschreiben stellte er fest, daß zur Ankurbelung der Wirtschaft die „Arbeitsbeschaffung zum normalen Lohn“ am wirksamsten sei. Der Arbeitsdienst hatte somit in den Augen Straßers nur sozialpädagogischen Wert, hingegen keine Bedeutung für die eigentliche Wirtschaftsankurbelung.

Das für den Reichstag bestimmte Antragsbündel verlangte staatliche Maßnahmen über einen Zeitraum von 4 Jahren. Antrag I forderte für Landeskulturarbeiten und ländliche Siedlung 3 Milliarden RM, aufgeteilt auf 4 Jahre. Antrag II sah zur Förderung des Eigenheimbaues 750 Millionen RM für die ersten beiden Jahre vor. Laut Antrag III sollten in den beiden ersten Jahren 600 Millionen RM zur Erneuerung und Erweiterung des Wege-, Straßen- und Kanalnetzes ausgegeben, laut Antrag IV 300 Millionen RM für Kredite an lebenswichtige Betriebe zur Ankurbelung der Produktion bereitgestellt werden. Was das Finanzierungsproblem anging, verwiesen

201 Brief des Reichsverbandes Deutscher Rundfunkteilnehmer an Straßer vom 25.6. 1932, BA, NS 22/3.

202 Rundfunkarchiv, Tonband VII, 20004/74 vom 14.6. 1932 und Tonband VII, 20004/86 vom 29.7. 1932.

203 Laut Auskunft des deutschen Rundfunkarchivs vom 25.10.1972 war Hitler als Rundfunkredner vorgesehen, doch beidemale wurde er kurzfristig von Gregor Straßer ersetzt.

204 Entwürfe für Wahlkampfmaterial, BA, NS 26/289.

205 Rundschreiben vom 24. 8. 1932 an alle NS-Fraktionen, BA, NS 22/348. 
die Anträge auf die Ersparnisse an Erwerbslosenunterstützung und auf die Mehreinnahmen an Steuern und Sozialbeiträgen. Der Rest der Finanzierung sollte durch „produktive Kreditschöpfung“ geschehen. Für die Länderparlamente waren die gleichen Anträge vorbereitet worden, nur daß hier die Höhe der Geldbeträge offenblieb, die sich nach den jeweiligen Bedingungen richten mußten. Da der Reichstag bereits wieder Neuwahlen entgegenging und nicht arbeitsfähig war, kam es hier nicht zur Einbringung dieser Anträge. Ähnlich war die Lage im Preußischen Landtag und in manchen anderen Landesparlamenten. Doch überall, wo es möglich war, gingen die NS-Fraktionen entsprechend den Straßerschen Entwürfen vor, wie etwa in Anhalt. Hier wurden im November 1932 Anträge zur Arbeitsbeschaffung durch die NS-Fraktion eingebracht, die dem Straßerschen Entwurf völlig entsprachen und zu deren Finanzierung die ,produktive Kreditschöpfung“ verstärkt gefordert wurde. ${ }^{206}$

Nach der Machtübernahme, als das Straßer-Programm nicht mehr namentlich erwähnt wurde, fußten denn auch die ersten Maßnahmen zur Ankurbelung der Wirtschaftskonjunktur, sowohl auf Gau- als auch auf Reichsebene, ${ }^{207}$ auf den Straßerschen Vorarbeiten, insbesondere der sogenannte „Reinhardt-Plan“, mit dem die Regierung Hitler am 1. Juni 1933 ihre eigene Wirtschaftspolitik begann. ${ }^{208}$ Als StraBer am 10. Mai 1932 sein Programm vor dem Reichstag verkündete, lag die Regierungsübernahme und damit die Möglichkeit, seine Pläne in die Wirklichkeit zu übersetzen, freilich noch in der Ferne. Umso bemerkenswerter ist daher ein kurzer Passus seiner Rede, der sich nicht eigentlich mit dem Wirtschaftsprogramm befaßte, innenpolitisch jedoch noch sensationeller wirken mußte, da er ein vorsichtig verkleidetes Angebot ausgerechnet an den ADGB enthielt. Daß eine Zusammenarbeit nur auf dem Boden der beiden Arbeitsbeschaffungsprogramme, nämlich des StraßerPlanes und des WTB-Planes der Gewerkschaften zustandekommen konnte, ließ Straßer deutlich durchblicken, wobei er allerdings verschwieg, daß beide Programme teilweise in Zusammenarbeit entstanden waren. ${ }^{209} \mathrm{Er}$ spielte sogar seine Kenntnis der Person Woytinskis, des wichtigsten Mitarbeiters des ADGB in der Studiengesellschaft und Mitschöpfers des gewerkschaftlichen Planes, ${ }^{210}$ herunter. $\mathrm{Zu}$ dem Gewerkschaftskongre $\beta$, auf dem dieser Plan angenommen worden war, sagte er: „Ich habe überhaupt den Eindruck, als ob dieser Gewerkschaftskongreß vielleicht deswe-

206 VB, 22. November 1932, S. 3.

207 Grotkopp, a. a. O., S. 283. Die Aktivitäten der Gauwirtschaftsberater und anderer Parteivertreter zusammen mit Wirtschaft und Verwaltung bewirkten, daß jene „Ausschöpfung privater Investitionsmöglichkeiten begonnen hatte, die in der Wirtschaft selbst aufgestaute Investitionen in Gang setzte." Vgl. Hans Kehrl, Krisenmanager im Dritten Reich, Düsseldorf 1973, S. $28 \mathrm{ff}$.

208 Reinhardt (bis Januar 1933 Reichspropagandaleiter II) bezeichnete sich im Sommer 1933 gegenüber Reichsfinanzminister Schwerin-Krosigk als Freund Straßers und erzählte, daß der nach ihm benannte Plan im wesentlichen auf Vorarbeiten Gregor Straßers beruhe. Auskunft Schwerin-Krosigk, v. 28. 11. 1971.

209 Siehe oben S. $105 \mathrm{ff}$.

210 Vgl. Kroll, a. a. O., S. $403 \mathrm{f}$. 
gen für das deutsche Volk schicksalhaft gewesen ist, weil er ein starkes Wiederaufleben der alten Tragik der deutschen Gewerkschaften in ihrem Gegensatz zur politischen, nach ganz anderen Gesichtspunkten handelnden Partei gebracht hat. Es ist eine Entwicklung, die unter Umständen in Deutschland eine Front schaffender Menschen im Rahmen der nationalen Selbsthilfe als möglich erscheinen läßt, ... Es ist doch ein wichtiges Zeichen, wenn in der Finanzierung des Arbeitsbeschaffungsprogramms der Gewerkschaften, über das man absolut reden kann, und bei dem wir jederzeit unter entsprechenden Bedingungen zur Mitarbeit bereit sind, die veraltete Führung zunächst keinen anderen Weg weiß als Kredite, Anleihen, Pumpen, und wenn dann in ihrer Zeitschrift „Die Arbeit“ ein Mann namens Woytinski, den ich persönlich nicht kenne, und von dem ich nicht weiß, ob er jung oder alt ist, diese Anleihen als Geldbeschaffungsmöglichkeit ablehnt und im weiteren Verlauf seiner Ausführungen zur Kreditschöpfung kommt, das heißt zu Finanzierungswegen, die letzten Endes von uns zum ersten Mal in die Debatte des deutschen Volkes geworfen worden sind." 211

Daraus ergibt sich die Frage, ob Straßer sein Programm dadurch in die Wirklichkeit umsetzen und an die Regierung kommen wollte, daß er unter Umständen zu Koalitionen bereit war, die in der damaligen politischen Landschaft Deutschlands fast revolutionär erscheinen mußten.

211 Verhandlungen des Reichstages, Bd. 446, S. 2512. 